\title{
Electronic Structure and Photoluminescence Properties of $\mathrm{Eu}\left(\eta^{9}-\mathrm{C}_{9} \mathrm{H}_{9}\right)_{2}$
}

\author{
Harry Ramanantoanina, ${ }^{* \dagger}{ }^{\dagger}$ Lynda Merzoud, ${ }^{\ddagger}$ Jules Tshishimbi Muya, ${ }^{\perp, \#}$ Henry Chermette, ${ }^{*, *}$ and Claude \\ Daul $^{*} \S$
}

${ }^{\dagger}$ Paul Scherrer Institute, CH-5232 Villigen PSI, Switzerland

Institut Sciences Analytiques, Universite de Lyon, Universite de Lyon 1, UMR CNRS 5280, 5 rue de la Doua, 69100 Villeurbanne, France

${ }^{\perp}$ Department of Chemistry, Hanyang University, 222 Wangsimni-ro, Seongdong-gu, Seoul 04763, Republic of Korea

\#

Department of Chemistry, Faculty of Sciences, University of Kinshasa, Kinshasa, DR Congo

$\S$

Department of Chemistry, University of Fribourg, CH-1700 Fribourg, Switzerland

Supporting Information

ABSTRACT: The electronic structure of $\mathrm{Eu}^{2+}$ compounds results from a complex combination of strongly correlated electrons and relativistic effects as well as weak ligand-field interaction. There is tremendous interest in calculating the electronic structure as nowadays the $\mathrm{Eu}^{2+}$ ion is becoming more and more crucial, for instance, in lighting technologies. Recently, interest in semiempirical methods to qualitatively evaluate the electronic structure and to model the optical spectra has gained popularity, although the theoretical methods strongly rely upon empirical inputs, hindering their prediction capabilities. Besides, ab initio multireference models are computationally heavy and demand very elaborative theoretical background. Herein, application of the ligand-field density functional theory (LFDFT) method that is recently available in the Amsterdam Modeling Suite is shown: (i) to elucidate the electronic structure properties on the basis of the multiplet

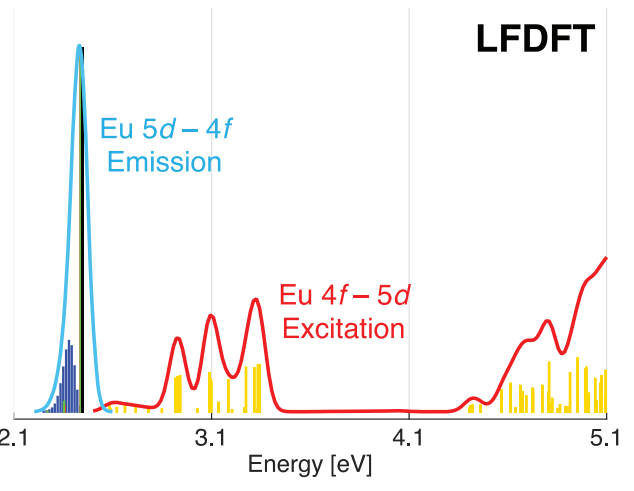
energy levels of Eu configurations $4 \mathrm{f}^{7}$ and $4 \mathrm{f}^{6} 5 \mathrm{~d}^{1}$ and (ii) to model the optical spectra quite accurately if compared to the conventional time-dependent density functional theory tool. We present a theoretical study of the molecular $\mathrm{Eu}\left(\eta^{9}-\mathrm{C}_{9} \mathrm{H}_{9}\right)_{2}$ complex and its underlying photoluminescence properties with respect to the Eu $4 \mathrm{f}-5 \mathrm{~d}$ electron transitions. We model the excitation and emission spectra with good agreement with the experiments, opening up the possibility of modeling lanthanides in complex environment like nanomaterials by means of LFDFT at much-reduced computational resources and cost.

\section{INTRODUCTION}

Lanthanide elements play an important role in many domains of chemical science, being useful in many applications, such as in functional materials, in biosensing and bioimaging, in catalysis, etc. ${ }^{1-5}$ The divalent europium ion $\left(\mathrm{Eu}^{2+}\right)$ is an example of a lanthanide ion that is nowadays in the forefront of inorganic chemistry research and development. ${ }^{6-10}$ In particular, $\mathrm{Eu}^{2+}$ compounds exhibit intense optical responses that result from the electric-dipole-allowed $4 \mathrm{f}-5 \mathrm{~d}$ electron transitions. Therefore, they are widely explored in lighting technologies and scintillators. ${ }^{6-10}$ The interest in coordination compounds with the $\mathrm{Eu}^{2+}$ ion has considerably increased in recent years, but calculation of their electronic structure properties still remains a veritable challenge.

Much attention has been also paid to the development of a theoretical model. In particular, the emergence of multireference methods including the configuration interaction algorithm $^{11-18}$ gives us the opportunity to elucidate the electronic structure from first-principles and predict the chemical reactivity and physical properties. However, existing theoretical models that are effectively adapted to deal with lanthanide coordination compounds are not trivial. These compounds are associated with strong electron correlations (obtained as per the open-shell $4 \mathrm{f}^{n}$ electron configuration) and relativistic effects; therefore, they still pose many questions in the field of applied computational chemistry. ${ }^{15,16}$

In this article, we focus on the calculation of the electronic structure of the $\mathrm{Eu}^{2+}$ ion and the description of the photoluminescence properties on the basis of the $4 f-5 d$ electron transitions. More precisely, we study the molecular 
$\mathrm{Eu}\left(\eta^{9}-\mathrm{C}_{9} \mathrm{H}_{9}\right)_{2}$ complex, motivated by the fact that (i) organometallic molecules have great potential as building blocks for functional nanomaterials, ${ }^{19}$ (ii) the experimental evidence of the structural and photoluminescence properties of $\mathrm{Eu}\left(\eta^{9}-\mathrm{C}_{9} \mathrm{H}_{9}\right)_{2}$ has been recently proposed, ${ }^{20}$ and (iii) our results can be used to assess the performance of the quantum chemistry code, ligand-field density functional theory (LFDFT), ${ }^{21-23}$ to predict complex optical spectra close to the experiments.

LFDFT is a density functional theory (DFT)-based model that is designed to incorporate the configuration interaction algorithm by means of a model Hamiltonian. ${ }^{21-23}$ With LFDFT, it is possible to calculate the electronic structure of lanthanide compounds and to predict the photophysical and magnetic properties. LFDFT is computationally economical and can be applied to any lanthanide system without limitation of molecular size or coordination symmetry. ${ }^{21-23}$ Therefore, it has a promising potential to characterize lanthanides in a complex environment, which may be difficult (if not impossible) to evaluate using existing theoretical methods. For instance, semiempirical methods are very popular, ${ }^{24-28}$ but they strongly rely on empirical inputs, hindering their prediction capabilities. Likewise, multireference methods like the complete active space self-consistent field (CASSCF) and related methodologies are also frequently applied and can give accurate results for large lanthanide complexes, ${ }^{11-18}$ although they are computationally heavy and demand a very elaborate theoretical background.

\section{METHODS}

Our studies require in the first place a structural investigation of the molecular $\mathrm{Eu}\left(\eta^{9}-\mathrm{C}_{9} \mathrm{H}_{9}\right)_{2}$ complex. The structural investigation consists of determining the stability of the complex in terms of total DFT energies as well as vibrational and frequency analysis. We expose two different starting geometries, i.e., the $\mathrm{Eu}^{2+}$ ion is coordinated with two cyclononatetraenyl $\left(\eta^{9}-\mathrm{C}_{9} \mathrm{H}_{9}\right)^{-}$ligands in $D_{9 h}$ and $D_{9 d}$ symmetries, respectively. Figure 1 shows graphically the

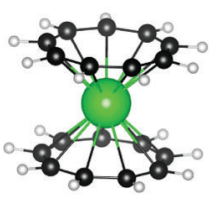

a

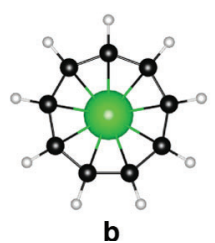

b

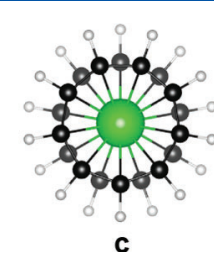

c
Figure 1. Ball-and-stick model of the molecular $\mathrm{Eu}\left(\eta^{9}-\mathrm{C}_{9} \mathrm{H}_{9}\right)^{2}$ complex (a) showing the structures that belong to the $D_{9 h}$ (b) and $D_{9 d}$ (c) point groups from the top view. Color code: Eu (in green), C (in black), and $\mathrm{H}$ (in gray).

molecular structures. We ideally optimize the geometry with the given high-symmetry constraint, but we also allow the descent in symmetry if a stable electronic structure (stationary point with a positive-definite Hessian) is not reached. All of the $\mathrm{Eu}$ 4f orbitals are singly occupied with electrons (spinunrestricted self-consistent field (SCF)), yielding a groundstate (GS) electronic structure derived from the ${ }^{8} \mathrm{~S}$ muliplet of the free $\mathrm{Eu}^{2+}$ ion.

With the most favorable geometry that is determined from the previous step, we calculate the electronic fine structure by using the LFDFT method. ${ }^{21-23}$ LFDFT works with a specific electron configuration system, operating a model Hamiltonian,
$H=H_{0}+H_{\mathrm{ER}}+H_{\mathrm{SO}}+H_{\mathrm{LF}}$, on top of DFT. The model Hamiltonian has four terms that denote the fine structure resolution that is neglected in the DFT calculation: the configuration average-energy correction $\left(H_{0}\right)$, interelectron repulsion $\left(H_{\mathrm{ER}}\right)$, spin-orbit coupling $\left(\mathrm{H}_{\mathrm{SO}}\right)$, and ligand-field splitting $\left(H_{\mathrm{LF}}\right)$. Recalling that LFDFT is recently available in the Amsterdam Density Functional (ADF) $\operatorname{code}^{29-31}$ a rigorous description of its methodology can be found elsewhere. ${ }^{32,33}$

However, it is noteworthy that we use the $\mathrm{ADF}^{29-31}$ because it allows rather nonstandard options that are important in the LFDFT methodology, like the selective occupation of molecular orbitals and the average of configuration (AOC)type calculation. ${ }^{34}$ This latter insures a statistically averaged electron density that will be isomorphic with the electron configuration system that forms the basis of the model Hamiltonian. In this work, we target two electron configuration systems: Eu configurations $4 \mathrm{f}^{7}$ and $4 \mathrm{f}^{6} 5 \mathrm{~d}^{1}$ that play important roles in the photoluminescence mechanism. ${ }^{6-10}$ The AOCtype calculation implies a spin-restricted SCF. ${ }^{32,33}$ Therefore, the molecular orbitals that are selectively identified with large fractional parentage coefficients for Eu $4 \mathrm{f}$ are occupied with 7/ $7=1$ electron, representing the Eu configuration $4 \mathrm{f}^{7}$. Similarly, the molecular orbitals that are selectively identified with large fractional parentage coefficients for $\mathrm{Eu} 4 \mathrm{f}$ and $5 \mathrm{~d}$, respectively, are occupied with $6 / 7=0.8571$ and $1 / 5=0.2000$ electrons, representing the Eu configuration $4 \mathrm{f}^{6} 5 \mathrm{~d}^{1}$. In $\mathrm{ADF},{ }^{29-31}$ the fractional parentage coefficients are listed in the output file along with the molecular orbital energies, and they represent the portion of the molecular orbitals with the most significant symmetrized fragment orbitals' gross population. ${ }^{29-31}$

The selective molecular orbitals form the active space of the LFDFT calculation, from which the matrix elements of the model Hamiltonian are constructed. In practice, we extract from DFT the configuration-average energy parameter, SlaterCondon integrals, spin-orbit coupling constants, and the ligand-field potential, whose quantities parametrize the model Hamiltonian. $^{32,33}$ The multiplet energy levels $E_{i}^{(0)}$ (with $i=1$, $2, \ldots, 3432)$ and $E_{k}^{(1)}($ with $k=1,2, \ldots, 30030)$ that arise from Eu configurations $4 \mathrm{f}^{7}$ and $4 \mathrm{f}^{6} 5 \mathrm{~d}^{1}$, respectively, as well as the associated multielectronic eigenfunctions $\psi_{i}^{(0)}$ and $\psi_{i}^{(1)}$ that are expressed as a function of single determinants of spin-orbitals are outputs of the LFDFT via full diagonalization of the matrix element of the model Hamiltonian. ${ }^{32,33}$

The photoluminescence properties are computed on the basis of the LFDFT outputs. That is, the excitation spectrum is obtained by calculating the oscillator strengths of the $4 \mathrm{f}^{7} \rightarrow$ $4 \mathrm{f}^{6} 5 \mathrm{~d}^{1}$ electron transitions. The initial and final states correspond to the GS multiplet level of $\mathrm{Eu} 4 \mathrm{f}^{7}$, without temperature effects, and the whole manifold of the multiplet levels of $\mathrm{Eu} 4 \mathrm{f}^{6} 5 \mathrm{~d}^{1}$, respectively. The emission spectrum involves vibronic coupling ${ }^{35-37}$ on top of the $4 f^{6} 5 \mathrm{~d}^{1} \rightarrow 4 f^{7}$ electron transitions, in that the initial and final states correspond to the lowest-energy multiplet of $\mathrm{Eu} 4 \mathrm{f}^{6} 5 \mathrm{~d}^{1}$ and the GS of Eu $4 \mathrm{f}^{7}$.

\section{COMPUTATIONAL DETAILS}

The present DFT results were obtained using the Local Density Approximation (LDA) Slater exchange and VoskoWilk-Nusair correlation, ${ }^{38}$ as well as the Generalized Gradient Approximation Perdew-Burke-Ernzerhof $(\mathrm{PBE})^{39}$ and the hybrid Becke's three parameters with the Lee-Yang-Parr correlation (B3LYP $)^{40}$ in the $\mathrm{ADF}$ suite of software. ${ }^{29-31}$ 
Furthermore, in order to check the importance of dispersion forces, we also used the Grimme corrections associated with the PBE functional in its D3 formulation and Becke-Johnson parametrization. $^{41}$

The molecular orbitals were expanded using the multiplezeta Slater-Type Orbitals (STO) functions with polarization extra functions, ${ }^{29-31}$ i.e., TZ2P for the Eu, C, and $\mathrm{H}$ atoms. The smaller TZP basis set has been also used for B3LYP calculations, leading to a negligible $D_{9 d}-D_{9 h}$ DFT energy difference with quasi-identical bond distances and bond angles (see Supporting Information Tables S1 and S2). The SCF was set up to take into consideration all electrons. This was necessary to take into account properly the $4 \mathrm{f}$ electrons, which, although being less involved in the bonding contributions of orbitals to the geometry, do play a role $(3.5 \%$ contribution here on top of the valence band molecular orbitals (below the 4f band) $).{ }^{42-44}$ The relativistic corrections were included via the Zeroth-Order Regular Approximation (ZORA) of the Dirac equation method. ${ }^{45}$ All calculations were done at the scalar ZORA relativistic level of theory, and spin-orbit coupling interaction was included by means of the spinorbit ZORA method. We did not included solvation modeling in our calculations because the solvent used in the experiment (toluene) is nonpolar. ${ }^{20}$ Indeed, we assumed that only polar solvents would lead to modified structures with respect to the gas phase.

\section{RESULTS AND DISCUSSION}

Structural Analysis. The compound has been isolated in the crystalline phase by Kawasaki et al., ${ }^{20}$ and the structure has been extracted from the Cambridge Crystallographic Data Center (CCSD) database. From the cif file, one can see on the one hand a $\left(\eta^{9}-\mathrm{C}_{9} \mathrm{H}_{9}\right)^{-}$ligand and on the other hand, symmetrically with respect to the Eu center, a superposition of two $\left(\eta^{9}-\mathrm{C}_{9} \mathrm{H}_{9}\right)^{-}$ligands leading to either a $D_{9 h}$ or a $D_{9 d}$ structure.

The two structures have been taken as inputs for geometry optimizations at the DFT levels (see the Methods section). These geometry optimizations in the gas phase led to quasidegenerate structures, as can be seen in Table 1. This

Table 1. Calculated Electronic Energies $\left(E^{\mathrm{DFT}}\right.$ in $\left.\mathrm{kJ} / \mathrm{mol}\right)$ Relative to the Global Minimum, Obtained from DFT Calculation of $\mathrm{Eu}\left(\eta^{9}-\mathrm{C}_{9} \mathrm{H}_{9}\right)_{2}$ with $D_{9 h}$ and $D_{9 d}$ Point Groups Together with the Lowest Calculated Vibrational Frequencies $\left(\hbar \omega\right.$ in $\left.\mathrm{cm}^{-1}\right)$

\begin{tabular}{ccccc} 
& & LDA & PBE & B3LYP \\
$D_{9 h}$ & $E_{\text {DFT }}$ & 0 & 0 & 0 \\
& $\hbar \omega_{1}$ & 12.3 & 16.4 & 48.8 \\
& $\hbar \omega_{2}$ & 49.7 & 47.8 & 62.0 \\
$D_{9 d}$ & $E^{\text {DFT }}$ & 0.002 & 0.06 & 0.63 \\
& $\hbar \omega_{1}$ & 15.6 & 18.2 & 46.3 \\
& $\hbar \omega_{2}$ & 50.4 & 48.0 & 56.0 \\
\hline
\end{tabular}

remarkable similarity of the energies of both conformations suggests excellent flexibility (and stability) of the complex. Therefore, we attempted to calculate the energy barrier between the $D_{9 h}$ and the $D_{9 d}$ structures through a linear transit calculation turning one $\left(\eta^{9}-\mathrm{C}_{9} \mathrm{H}_{9}\right)^{-}$ligand by a $2^{\circ}$ step around the $C_{9}$ axis. We did not find a transition state. Indeed, with the evolution of the electronic energy along the linear transit, the energy fluctuation along the rotation was just some wiggling of an energy potential surface fully planar. Accordingly, these energies led us to think that the ligands are allowed to quasi-freely rotate in the gas phase and to be stabilized by dispersion (packing) forces in the crystalline phase. Therefore, the experimental observables at least at room temperature result from the convolution of the properties of both the $D_{9 d}$ and $D_{9 h}$ structures with some Gaussian. Indeed, looking more deeply at the structure data in the CCDC, we could see that the geometry given in the cif file results from a constrained refinement, but indeed, with less constraints, one should have toric volumes instead of spherical atoms.

Because of the tiny energy difference between the $D_{9 h}$ and $D_{9 d}$ structures, we performed supplementary calculations with increased numerical precision when possible: the TZ2P basis set (triple- $\zeta$ plus two polarization functions) was used for LDA, PBE, and B3LYP functionals This basis set being already large, the numerical accuracy was also improved at the PBE level with more dense numerical integration grids: good, verygood, and excellent (in ADF terminology, ${ }^{29-31}$ as well as "boost 3" for the radial integration grid). The results do not show any significant deviation, underlining the stability of our calculation (see Supporting Information Tables S1 and S2). From the crystalline structure obtained at $103 \mathrm{~K}$, Kawasaki et al. ${ }^{20}$ noticed a significant disorder on the $\left(\eta^{9}-\mathrm{C}_{9} \mathrm{H}_{9}\right)^{-}$ring, and they obtained a ratio of $58 / 42$ for $D_{9 h} / D_{9 d}$, in agreement with a free rotation of the $\left(\eta^{9}-\mathrm{C}_{9} \mathrm{H}_{9}\right)^{-}$ring and therefore with our calculations. Indeed, from the ratio 58/42, we can estimate the equilibrium constant to 1.38 , leading to an energy difference $R T \ln (K)$ amounting to $0.8 \mathrm{~kJ} / \mathrm{mol}$ at room temperature and $0.27 \mathrm{~kJ} / \mathrm{mol}$ at $103 \mathrm{~K}$. This is fully in agreement with out PBE calculation (Table 1).

Table 2 lists calculated geometrical parameters of $\mathrm{Eu}\left(\eta^{9}\right.$ $\left.\mathrm{C}_{9} \mathrm{H}_{9}\right)_{2}$ in $D_{9 h}$ (see also Supporting Information Table S1 for

Table 2. Calculated Geometrical Parameters for $\mathrm{Eu}\left(\boldsymbol{\eta}^{9}\right.$ $\left.\mathrm{C}_{9} \mathrm{H}_{9}\right)_{2}$ in the Ground Eu Configuration $4 \mathrm{f}^{7}$ (GS) within the $D_{9 d}$ Point Group ${ }^{a}$

\begin{tabular}{lcccc}
\multicolumn{1}{c}{$D_{9 h}$} & LDA & PBE & B3LYP & exp. $^{b}$ \\
$d(\mathrm{Eu}-\mathrm{C})$ & 2.779 & 2.887 & 2.956 & 2.900 \\
$d(\mathrm{C}-\mathrm{X})^{c}$ & 2.039 & 2.057 & 2.048 & 2.036 \\
$d(\mathrm{C}-\mathrm{H})$ & 1.095 & 1.093 & 1.084 & 0.950 \\
$d(\mathrm{Eu}-\mathrm{X})^{c}$ & 1.888 & 2.026 & 2.131 & 2.065 \\
$A(\mathrm{X}-\mathrm{C}-\mathrm{H})^{c}$ & 176.6 & 177.1 & 177.7 & 178.9
\end{tabular}

${ }^{a}$ Bond distances are $d$ in $\AA$, and bond angles are $A$ in deg. ${ }^{b}$ Average values taken from ref $20{ }^{c} \mathrm{X}$ stands for the centroid of the $\left(\eta^{9}-\mathrm{C}_{9} \mathrm{H}_{9}\right)^{-}$ ring.

the $D_{9 d}$ structure). Taking into account the geometries obtained at the PBE/TZ2P level with excellent numerical accuracy, the carbon atoms are evidently (because of the $D_{9 h}$ and $D_{9 d}$ symmetries) located at the same distances from the Eu atom (see Table 2 and Supporting Information Table S1). At the same level of theory, an $\mathrm{Eu}-\mathrm{X}$, with $\mathrm{X}$ standing for the centroid of the $\left(\eta^{9}-\mathrm{C}_{9} \mathrm{H}_{9}\right)^{-}$ring, distance of $2.026 \AA$ is obtained in both symmetries, a result that is not imposed by the symmetry (such as, for instance, the $\mathrm{X}-\mathrm{Eu}-\mathrm{X}$ angle of $180^{\circ}$ ). The $\mathrm{Eu}-\mathrm{X}$ distance is larger by $0.14 \AA$ than the LDA distance, smaller by $0.11 \AA$ than the B3LYP distance (Table 2), and close to the experimental one (1.99-2.09).

Including dispersion forces with the PBE-D3(BJ) functional does not significantly influence distances. The variation of the $\mathrm{X}-\mathrm{C}-\mathrm{H}$ angle is also estimated with the different calculation 
models. The absolute deviations of the PBE/TZ2P level with excellent numerical accuracy data with respect to LDA- and B3LYP-calculated $\mathrm{X}-\mathrm{C}-\mathrm{H}$ angles are 0.5 and $0.6^{\circ}$, respectively. However, adding the effects of dispersion to the PBE functional with the $\mathrm{B} 3(\mathrm{DJ})$ corrections leads to changes of only $0.3^{\circ}$.

Kawasaki et al. ${ }^{20}$ performed DFT calculations at the B3LYP level but with a large core effective potential that could not take into account the semicore $4 \mathrm{f}$ orbitals, which are rather contracted with respect to $5 \mathrm{~d}$ orbitals but energetically high. Our vibrational analysis does not lead to significant differences with respect to their work; ${ }^{20}$ therefore, we will not discuss it more. Nevertheless, the low frequencies at the PBE level are found to be slightly smaller than the B3LYP ones. We report in Table 1 these lower frequencies, and we look at the corresponding modes: it is gratifying to see that the lowest mode $\left(15 \mathrm{~cm}^{-1}\right)$ is related to the (quasi-)free rotation of the $\left(\eta^{9}-\mathrm{C}_{9} \mathrm{H}_{9}\right)^{-}$rings around the $C_{9}$ axis, whereas the second lowest frequencies $\left(50 \mathrm{~cm}^{-1}\right)$ are related to a wagging mode between the two $\left(\eta^{9}-\mathrm{C}_{9} \mathrm{H}_{9}\right)^{-}$planes (graphical representations of these vibrational modes are given in Supporting Information Figure S1). This low mode allows the deformation of the complex to be easy even at low temperature and is responsible for the scattering of the experimental $\mathrm{Eu}-\mathrm{C}$ distances (averages of $2.84 \AA$ (2.80-2.90 range) and $2.90 \AA$ (2.862.96 range)).

Molecular Orbital Diagram. On the basis of the results described in the previous section, we have decided to set the structure of $\mathrm{Eu}\left(\eta^{9}-\mathrm{C}_{9} \mathrm{H}_{9}\right)_{2}$ to the $D_{9 h}$ symmetry. Therefore, we continue the investigation with this conformation using the equilibrium structures obtained from DFT with the LDA, PBE, and B3LYP functionals (Table 2). Note that we found that the fluctuation of the local symmetry among the $D_{9 h}, D_{9}$, and $D_{9 d}$ does not bring significant changes in our LFDFT results. ${ }^{46}$

Because the nine-fold dihedral point groups $D_{9 d}, D_{9 h}$ and $D_{9}$ are rather rare in coordination chemistry, Table 3 shows all of the irreducible representations (irreps) that form the basis of the valence orbitals of the $\mathrm{Eu}$ and $\mathrm{C}$ atoms in the molecular $\mathrm{Eu}\left(\eta^{9}-\mathrm{C}_{9} \mathrm{H}_{9}\right)_{2}$ complex, as well as the corresponding basis functions for all of the relevant metal and ligand orbitals. Under $D_{9 h}$, the degeneracy of the $\mathrm{Eu} 4 \mathrm{f}$ orbitals is lifted, forming four components that span the $\mathrm{a}_{2}{ }^{\prime \prime}, \mathrm{e}_{1}{ }^{\prime}, \mathrm{e}_{2}{ }^{\prime \prime}$, and $\mathrm{e}_{3}{ }^{\prime}$

Table 3. Symmetry transformation of the Eu 4f, $5 \mathrm{~d}$ and $\mathrm{C} 2 p$ atomic orbitals in the molecular $\mathrm{Eu}\left(\eta^{9}-\mathrm{C}_{9} \mathrm{H}_{9}\right)_{2}$ complex within the $D_{9}, D_{9 h}$ and $D_{9 d}$ point groups

\begin{tabular}{|c|c|c|c|}
\hline & $D_{9}$ & $D_{9 h}$ & $D_{9 d}$ \\
\hline $\begin{array}{l}\mathrm{Eu} \\
4 \mathrm{f}_{\sigma}\end{array}$ & $a_{2}$ & $a_{2}{ }^{\prime \prime}$ & $a_{2 u}$ \\
\hline $\mathrm{Eu}_{4 \mathrm{f}_{\pi}}$ & $\mathrm{e}_{1}$ & $e_{1}^{\prime}$ & $\mathrm{e}_{1 \mathrm{u}}$ \\
\hline $\mathrm{Eu}_{\delta}$ & $e_{2}$ & $\mathrm{e}_{2}^{\prime \prime}$ & $e_{2 u}$ \\
\hline $\mathrm{Eu}_{4 \mathrm{f}_{\varphi}}$ & $e_{3}$ & $e_{3}^{\prime}$ & $e_{3 u}$ \\
\hline $\begin{array}{l}\mathrm{Eu} \\
5 \mathrm{~d}_{\sigma}\end{array}$ & $a_{1}$ & $a_{1}{ }^{\prime}$ & $a_{1 g}$ \\
\hline $\mathrm{Eu}_{5}$ & $\mathrm{e}_{1}$ & $\mathrm{e}_{1}^{\prime \prime}$ & $e_{1 g}$ \\
\hline $\mathrm{Eu}_{5 \mathrm{~d}_{\delta}}$ & $\mathrm{e}_{2}$ & $\mathrm{e}_{2}^{\prime}$ & $e_{2 g}$ \\
\hline $\mathrm{C}_{2 \mathrm{p}_{\pi}}$ & $\begin{array}{c}\mathrm{a}_{1}+\mathrm{a}_{2}+2 \mathrm{e}_{1}+ \\
2 \mathrm{e}_{2}+2 \mathrm{e}_{3}+ \\
2 \mathrm{e}_{4}\end{array}$ & $\begin{array}{l}\mathrm{a}_{1}{ }^{\prime}+\mathrm{a}_{2}^{\prime \prime}+\mathrm{e}_{1}^{\prime}+\mathrm{e}_{1}^{\prime \prime}+\mathrm{e}_{2}^{\prime} \\
\quad+\mathrm{e}_{2}^{\prime \prime}+\mathrm{e}_{3}^{\prime}+\mathrm{e}_{3}^{\prime \prime}+\mathrm{e}_{4}^{\prime} \\
\quad+\mathrm{e}_{4}^{\prime \prime}\end{array}$ & $\begin{array}{l}a_{1 g}+a_{2 u}+e_{1 g}+e_{1 u}+e_{2 g} \\
\quad+e_{2 u}+e_{3 g}+e_{3 u}+e_{4 g} \\
\quad+e_{4 u}\end{array}$ \\
\hline
\end{tabular}

irreps. These components correspond to functions with $4 \mathrm{f}_{z^{3}}$, $\left(4 \mathrm{f}_{z^{2} x}, 4 \mathrm{f}_{z^{2} y}\right),\left(4 \mathrm{f}_{x y z}, 4 \mathrm{f}_{z\left(x^{2}-y^{2}\right)}\right)$, and $\left(4 \mathrm{f}_{x\left(x^{2}-3 y^{2}\right)}, 4 \mathrm{f}_{y\left(3 x^{2}-y^{2}\right)}\right)$, respectively.

The ligand-field is comparable in general to the ordering of molecular orbitals in a linear $C_{\infty \nu}$ point group, with $\sigma, \pi, \delta$, and $\varphi$ corresponding to $z^{3},\left(z^{2} x, z^{2} y\right),\left(x y z, z\left(x^{2}-y^{2}\right)\right)$, and $\left(x\left(x^{2}\right.\right.$ $\left.\left.-3 y^{2}\right), y\left(3 x^{2}-y^{2}\right)\right)$, respectively. In fact, the ligand-field splitting of $\mathrm{Eu} 4 \mathrm{f}$ and $5 \mathrm{~d}$ orbitals preserve $m_{l}$ as a good quantum number in $D_{9 h}$ (respectively in $D_{9 d}$ and $D_{9}$ ) symmetry, the axis of quantization being parallel to the $C_{9}$ axis of the $\mathrm{Eu}\left(\eta^{9}-\mathrm{C}_{9} \mathrm{H}_{9}\right)_{2}$ structure. Therefore, the notation $4 \mathrm{f}_{\sigma}$, $4 \mathrm{f}_{\pi}, 4 \mathrm{f}_{\delta}$, and $4 \mathrm{f}_{\varphi}$ will be used, for simplicity, in the discussion to express the four components of ligand-field splitting (see Table 3). Similarly, the degeneracy of the Eu 5d orbitals is also lifted under $D_{9 h}$ symmetry, resulting in three components with $\mathrm{a}_{1}{ }^{\prime}\left(5 \mathrm{~d}_{z}{ }^{2}\right), \mathrm{e}_{1}{ }^{\prime}\left(5 \mathrm{~d}_{x z}, 5 \mathrm{~d}_{y z}\right)$, and $\mathrm{e}_{2}{ }^{\prime}\left(5 \mathrm{~d}_{x y}, 5 \mathrm{~d}_{x^{2}-y^{2}}\right)$ representations. They will also be represented using the expression $5 \mathrm{~d}_{\sigma}$, $5 \mathrm{~d}_{\pi}$, and $5 \mathrm{~d}_{\delta}$ of the linear point group, with $\sigma, \pi$, and $\delta$ corresponding to $z^{2},(x z, y z)$, and $\left(x y, x^{2}-y^{2}\right)$, respectively.

Figure 2 shows the calculated molecular orbital diagram of $\mathrm{Eu}\left(\eta^{9}-\mathrm{C}_{9} \mathrm{H}_{9}\right)_{2}$ as a result of the interaction between the $\mathrm{Eu} 4 \mathrm{f}$

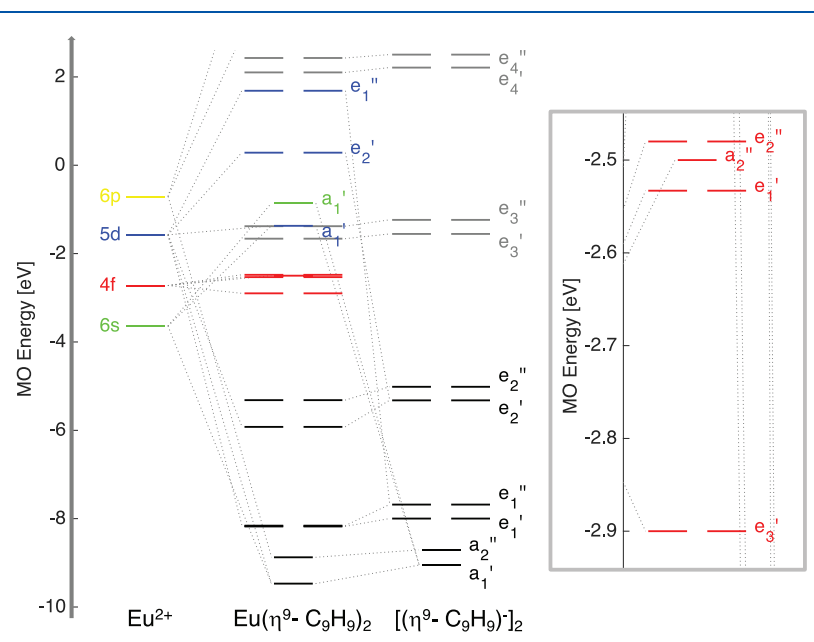

Figure 2. Representation of the molecular orbital diagram (bars) of $\mathrm{Eu}\left(\eta^{9}-\mathrm{C}_{9} \mathrm{H}_{9}\right)_{2}$ in $D_{9 h}$ symmetry with Eu configuration $4 \mathrm{f}^{7}$ (DFT calculation using the PBE functional), showing the interactions among Eu 4f, 5d, and $6 \mathrm{~s}$ with $\mathrm{C} 2 \mathrm{p}_{\pi}$. The energy range corresponding to the ligand-field splitting of the Eu $4 \mathrm{f}$ are highlighted (inset). In that, the energy of the $a_{2}{ }^{\prime \prime}$ orbital is deliberately shifted for nicer illustration (see also Table 4 for the correct value). Color code: see the text for details.

and $5 \mathrm{~d}$ with the $\mathrm{C} 2 \mathrm{p}_{\pi}$ of the ligands. The $C 2 \mathrm{p}_{\pi}$ orbitals of the parallel and planar $\left[\left(\eta^{9}-\mathrm{C}_{9} \mathrm{H}_{9}\right)^{-}\right]_{2}$ ligands form the basis of 18 $\pi$ molecular orbitals that are denominated with the irreps listed in Table 3. In order of increasing energy, we obtain the following: $\mathrm{a}_{1}{ }^{\prime}<\mathrm{a}_{2}{ }^{\prime \prime}<\mathrm{e}_{1}{ }^{\prime}<\mathrm{e}_{1}{ }^{\prime \prime}<\mathrm{e}_{2}{ }^{\prime}<\mathrm{e}_{2}{ }^{\prime \prime}<\mathrm{e}_{3}{ }^{\prime}<\mathrm{e}_{3}{ }^{\prime \prime}<\mathrm{e}_{4}{ }^{\prime}<$ $\mathrm{e}_{4}^{\prime \prime}$, where up to the $\mathrm{e}_{2}^{\prime}$ level (represented with black bars in Figure 2) the molecular orbitals are fully occupied with electrons (20 in total). The remaining molecular orbitals with $\mathrm{e}_{3}{ }^{\prime}, \mathrm{e}_{3}{ }^{\prime \prime}, \mathrm{e}_{4}{ }^{\prime}$, and $\mathrm{e}_{4}{ }^{\prime \prime}$ irreps (represented with gray bars in Figure 2) are empty.

The results of the DFT calculations of $\mathrm{Eu}\left(\eta^{9}-\mathrm{C}_{9} \mathrm{H}_{9}\right)_{2}$ with Eu configuration $4 \mathrm{f}^{7}$ are summarized in Table 4 . The selective molecular orbital energies are listed together with the electron occupation scheme imposed in DFT and the parentage coefficients of the molecular complex from $\mathrm{Eu} 4 \mathrm{f}$ atomic 
Table 4. Selective Kohn-Sham Molecular Orbital Energy Levels $\left(E_{4 \mathrm{f}}^{\mathrm{DFT}}\right.$ in eV) of the Molecular Eu $\left(\eta^{9}-\mathrm{C}_{9} \mathrm{H}_{9}\right)_{2} \mathrm{Complex}$ in the

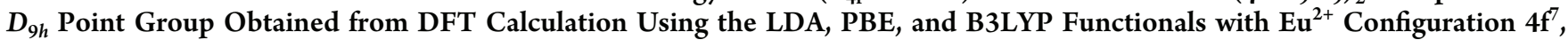
Showing Also the Occupation Scheme (ccc. in [-]) Imposed in the DFT Calculation and the Parentage Coefficients (coef. in $\%)$ of $\mathrm{Eu}\left(\eta^{9}-\mathrm{C}_{9} \mathrm{H}_{9}\right)_{2}$ from Eu 4f Atomic Orbitals

\begin{tabular}{|c|c|c|c|c|c|c|c|}
\hline $\mathrm{Eu}$ & & LDA & & $\mathrm{PBE}$ & & B3LYP & \\
\hline $4 f^{7}$ & occ. & $E_{4 \mathrm{f}}^{\mathrm{DFT}}$ & coef. & $E_{4 \mathrm{f}}^{\mathrm{DFT}}$ & coef. & $E_{4 \mathrm{f}}^{\mathrm{DFT}}$ & coef. \\
\hline$e_{3}^{\prime}$ & 2 & -3.2116 & $87.64 \mathrm{f}_{\varphi}$ & -2.8991 & $90.44 \mathrm{f}_{\varphi}$ & -2.6698 & $91.24 \mathrm{f}_{\varphi}$ \\
\hline $\mathrm{e}_{1}{ }^{\prime}$ & 2 & -2.7344 & $99.24 \mathrm{f}_{\pi}$ & -2.5322 & $99.54 \mathrm{f}_{\pi}$ & -2.5184 & $99.04 \mathrm{f}_{\pi}$ \\
\hline$a_{2}{ }^{\prime \prime}$ & 1 & -2.7202 & $98.84 \mathrm{f}_{\sigma}$ & -2.5323 & $99.14 \mathrm{f}_{\sigma}$ & -2.5296 & $99.24 \mathrm{f}_{\sigma}$ \\
\hline $\mathrm{e}_{2}^{\prime \prime}$ & 2 & -2.6453 & $95.64 \mathrm{f}_{\delta}$ & -2.4794 & $96.74 \mathrm{f}_{\delta}$ & -2.5368 & $96.54 \mathrm{f}_{\delta}$ \\
\hline
\end{tabular}

Table 5. Selective Kohn-Sham Molecular Orbital Energy Levels $\left(E_{4 \mathrm{f}}^{\mathrm{DFT}}\right.$ and $E_{5 \mathrm{~d}}^{\mathrm{DFT}}$ in eV) of the Molecular $\mathrm{Eu}\left(\eta^{9}-\mathrm{C}_{9} \mathrm{H}_{9}\right)_{2}$ Complex in the $D_{9 h}$ Point Group Obtained from DFT Calculation Using the LDA, PBE, and B3LYP Functionals with Eu ${ }^{2+}$ Configuration $4 f^{6} 5 d^{1}$, Showing Also the Occupation Scheme (occ. in [-]) Imposed in the DFT Calculation and the Parentage Coefficients (coef. in \%) of $\mathrm{Eu}\left(\eta^{9}-\mathrm{C}_{9} \mathrm{H}_{9}\right)_{2}$ from $\mathrm{Eu} 4 \mathrm{f}$ and $5 \mathrm{~d}$ Atomic Orbitals

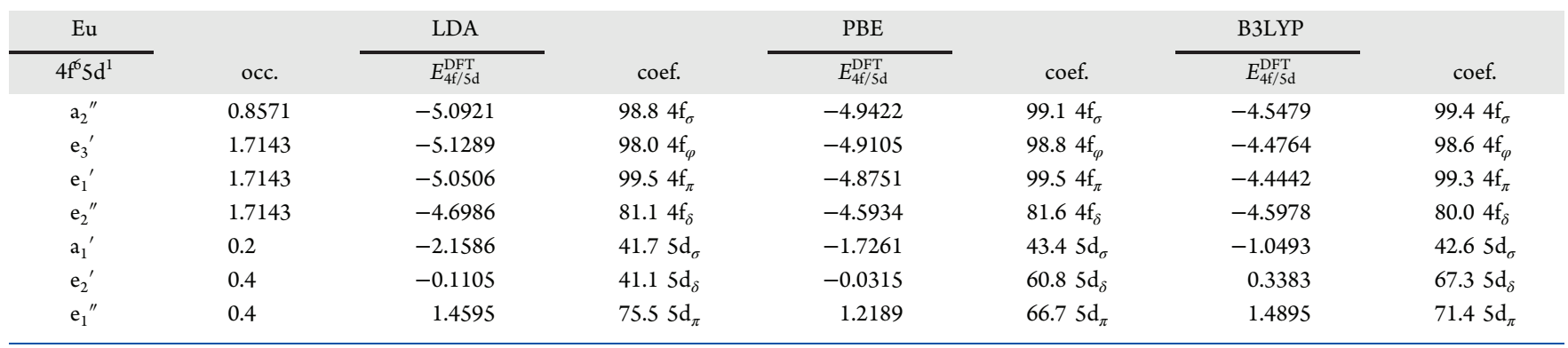

orbitals. The ligand-field splitting of the Eu $4 \mathrm{f}$ orbitals follows the sequence $\mathrm{e}_{3}{ }^{\prime}\left(4 \mathrm{f}_{\varphi}\right)<\mathrm{e}_{1}{ }^{\prime}\left(4 \mathrm{f}_{\pi}\right)<\mathrm{a}_{2}{ }^{\prime \prime}\left(4 \mathrm{f}_{\sigma}\right)<\mathrm{e}_{2}{ }^{\prime \prime}\left(4 \mathrm{f}_{\delta}\right)$ (represented with the red bars in Figure 2) as results of DFT using the LDA and PBE functionals. We observe that $\mathrm{e}_{3}{ }^{\prime}$ has the lowest energy independent of the choice of DFT functional (Table 4) because the $4 \mathrm{f}_{\varphi}$ orbitals have a small interaction with the molecular orbitals of the ligands. In addition, the $4 \mathrm{f}_{\varphi}$ orbitals are also stabilized by moderate interaction with the LUMO of the $\left[\left(\eta^{9}-\mathrm{C}_{9} \mathrm{H}_{9}\right)^{-}\right]_{2}$ ligands that share the same $\mathrm{e}_{3}{ }^{\prime}$ representation in the $D_{9 h}$ point group (see Figure 2). The LDA and $\mathrm{PBE}$ results show that the $\mathrm{e}_{2}{ }^{\prime \prime}$ is the most destabilized in energy (Table 4), indicating a strong interaction between the $4 \mathrm{f}_{\delta}$ orbitals with the HOMO of the $\left[\left(\eta^{9}-\mathrm{C}_{9} \mathrm{H}_{9}\right)^{-}\right]_{2}$ ligands. However, we admit that the B3LYP results are a little counterintuitive because we established a different assumption, the $\mathrm{e}_{1}{ }^{\prime}$ being the most destabilized molecular orbital energy (see Table 4). We did not find relevant studies of the electronic structure of $\mathrm{Eu}\left(\eta^{9}-\mathrm{C}_{9} \mathrm{H}_{9}\right)_{2}$ addressed with the help of existing theoretical models. While at this point, let us mention the recent studies in ref 47 that reported the synthesis and electronic structure of $\operatorname{Ln}\left(\eta^{9}-\mathrm{C}_{9} \mathrm{H}_{9}\right)_{2}$, with $\mathrm{Ln}=\mathrm{Yb}, \mathrm{Sm}$, and $\mathrm{Tm}$. However, we can compare our results to systems with the ligand $\left(\eta^{8}-\mathrm{C}_{8} \mathrm{H}_{8}\right)^{2-}$ that is isoelectronic with the present $\left(\eta^{9}-\mathrm{C}_{9} \mathrm{H}_{9}\right)^{-}$. Many experimental and theoretical studies of actinide and lanthanide sandwich complexes with $\left(\eta^{8}-\mathrm{C}_{8} \mathrm{H}_{8}\right)^{2-}$ are found in the open literature. ${ }^{48-55}$

The molecular $\mathrm{U}\left(\eta^{8}-\mathrm{C}_{8} \mathrm{H}_{8}\right)_{2}$ complex is, for instance, an example of a system that has been extensively investigated. $^{49,51,52}$ The spin-orbit configuration interaction model in ref 49 as well as the DFT calculation in refs 51 and 52 allow identification of the following sequence: $\left(5 f_{\sigma}\right)<\left(5 f_{\varphi}\right)<\left(5 f_{\pi}\right)<$ $\left(5 \mathrm{f}_{\delta}\right)$ of the ligand-field splitting of $\mathrm{U} 5 \mathrm{f}$ orbitals. These results support our LDA and PBE findings, although the chemistry of the $4 \mathrm{f}$ and $5 f$ orbitals are susceptible to variation. A periodic trend in the magnetism of $\left[\mathrm{Ln}\left(\eta^{8}-\mathrm{C}_{8} \mathrm{H}_{8}\right)_{2}\right]^{-}$with trivalent lanthanide ions as well as a trend in the nonlinear optical properties is proposed in refs 53 and 56. The electronic structures are determined from the CASSCF calculation. ${ }^{53}$ Stevens parameters are also reported for the representation of the ligand-field potential, ${ }^{53}$ in which only the $B_{2}^{0}, B_{4}^{0}$, and $B_{6}^{0}$ terms do not vanish by symmetry. By reconstructing the ligand-field potential from these terms, the periodic trend of the lanthanide $4 \mathrm{f}$ ligand-field splitting follows $\left(4 \mathrm{f}_{\varphi}\right)<\left(4 \mathrm{f}_{\pi}\right)<$ $\left(4 \mathrm{f}_{\sigma}\right)<\left(4 \mathrm{f}_{\delta}\right)$, corroborating also the LDA and PBE results. Eventually, the electronic structure of the isostructural $\operatorname{Ln}\left(\eta^{9}\right.$ $\left.\mathrm{C}_{9} \mathrm{H}_{9}\right)_{2}$, with $\mathrm{Ln}=\mathrm{Yb}, \mathrm{Sm}$, and $\mathrm{Tm},{ }^{47}$ allows one to also obtain the sequence $\left.4 \mathrm{f}_{\varphi}\right)<\left(4 \mathrm{f}_{\pi}\right)<\left(4 \mathrm{f}_{\sigma}\right)<\left(4 \mathrm{f}_{\delta}\right)$, in line with the LDA and PBE results.

The calculated parentage coefficients of $\mathrm{Eu}\left(\eta^{9}-\mathrm{C}_{9} \mathrm{H}_{9}\right)_{2}$ from $\mathrm{Eu} 4 \mathrm{f}$ (Table 4) show that the listed molecular orbitals are mainly of atomic character, independent of the choice of the DFT functional. We obtain the percentage coefficients that are in general larger than $90 \%$, the listed orbitals being well-suited to resolve the ligand-field states of the Eu configuration $4 \mathrm{f}^{7}$. Furthermore, the bonding interaction between the $\mathrm{Eu} 4 \mathrm{f}$ orbitals and the $\left(\eta^{9}-\mathrm{C}_{9} \mathrm{H}_{9}\right)^{-}$ligand is relatively weak, in agreement with the general trend in the bonding regime of lanthanide complexes that are well-known from previous studies. ${ }^{53,56}$ In fact, we observe that the lanthanide-ligand chemical bonding inclines heavily toward the $6 \mathrm{~s}$ and $5 \mathrm{~d}$ orbitals, although the role of the $4 \mathrm{f}$ cannot be neglected in some cases. ${ }^{42-44,55,56}$

The results of the DFT calculations of $\mathrm{Eu}\left(\eta^{9}-\mathrm{C}_{9} \mathrm{H}_{9}\right)_{2}$ with Eu configuration $4 f^{6} 5 d^{1}$ are shown in Table 5. Table 5 lists selective molecular orbital energies, together with the electron occupation schemes that are imposed in DFT and the parentage coefficients of the molecular complex from Eu $4 \mathrm{f}$ and $5 \mathrm{~d}$ atomic orbitals. The ligand-field splitting of the $\mathrm{Eu} 5 \mathrm{~d}$ orbitals follows the sequence $\mathrm{a}_{1}{ }^{\prime}\left(5 \mathrm{~d}_{\sigma}\right)<\mathrm{e}_{2}{ }^{\prime}\left(5 \mathrm{~d}_{\delta}\right)<\mathrm{e}_{1}{ }^{\prime}\left(5 \mathrm{~d}_{\pi}\right)$ (also represented with the blue bars in Figure 2 ), the qualitative picture being, as well, independent to the choice of the DFT functional. 
The order of the Eu 5d ligand-field is comparable to that obtained for $\mathrm{U} 6 \mathrm{~d}$, if compared to the previous study of the $\mathrm{U}\left(\eta^{8}-\mathrm{C}_{8} \mathrm{H}_{8}\right)_{2}$ complex. $^{49,52}$ However, the $5 \mathrm{~d}$ energy splitting is smaller (circa $2 / 3$ of the value obtained for $6 \mathrm{~d}$ ). The Eu $5 \mathrm{~d}_{\pi}$ and $5 \mathrm{~d}_{\delta}$ orbitals are particularly destabilized through strong interaction with the ligand orbitals. We note also that the ligand-field splitting of the Eu 5d orbitals is 30 times larger than that obtained for $\mathrm{Eu} \mathrm{4f,} \mathrm{the} \mathrm{Eu} 5 \mathrm{~d}$ being more available for bonding properties. ${ }^{15,57}$

The calculated parentage coefficients of $\mathrm{Eu}\left(\eta^{9}-\mathrm{C}_{9} \mathrm{H}_{9}\right)_{2}$ from Eu $5 d$ are relatively weak (see Table 5). Namely, we obtained circa $43 \%$ for $5 d_{\sigma}$. Note that two molecular orbitals share the same $\mathrm{a}_{1}{ }^{\prime}$ representation in the energy range close to the Eu $5 \mathrm{~d}$ ligand-field splitting (represented with the green and blue bars in Figure 2). In these two molecular orbitals, the $\mathrm{Eu} 5 \mathrm{~d}_{\sigma}$ and $6 \mathrm{~s}$ are partly hybridized together with some ligand contribution. While empty, the fractions of Eu $5 \mathrm{~d}_{\sigma}$ and $6 \mathrm{~s}$ of the two molecular orbitals are somewhat equivalent as results of the DFT calculation of the molecular complex with the Eu $4 \mathrm{f}^{7}$ configuration. By selectively occupying the molecular orbital with an electron, like for the calculation with Eu configuration $4 f^{6} 5 d^{1}$, the fractions of $\mathrm{Eu} 5 \mathrm{~d}_{\sigma}$ or $6 \mathrm{~s}$ become predominant.

Still considering the parentage coefficients of $\mathrm{Eu}\left(\eta^{9}-\mathrm{C}_{9} \mathrm{H}_{9}\right)_{2}$ from Eu 5d (Table 5), it is clear that the Eu 5d are essentially responsible for the bonding interaction in the molecular complex, in line with previous studies of lanthanide compounds. ${ }^{15,57}$ The reduced fraction of $\mathrm{Eu} 5 \mathrm{~d}$ supposes that the molecular orbitals contain a non-negligible amount of ligand. It is then clear that we use molecular orbitals that are strongly mixed with the ligands for the Eu 5d ligand-field states. This is not a problem itself because in ligand-field theory the nephelauxetic effect accounts for this specific situation. ${ }^{58,59}$ Although the nephelauxetic effect implies partial reduction of the Slater-Condon integrals from the free ion to the molecular complex in the semiempirical ligand-field concept, ${ }^{58}$ we do not perform any corrections or scaling factors in our LFDFT calculation, all of the parameters being extracted from first-principles by means of the DFT calculation.

The energies of the molecular orbitals that have large Eu $4 f$ fractional parentage coefficients vary from the results with $\mathrm{Eu}$ configuration $4 \mathrm{f}^{7}$ (Table 4) to that with $4 \mathrm{f}^{6} 5 \mathrm{~d}^{1}$ (Table 5). There is a shift of the molecular orbital energies toward lower values and a flip of the order of the molecular orbitals. We suppose that the supplementary hole induced in the $4 \mathrm{f}$ shell is a reason that certainly induces the change, leading to a situation where a screening effect ensures an overall stabilization of the molecular orbital energies. However, also, the $4 f$ orbitals become more contracted in configuration $4 f^{6} 5 \mathrm{~d}^{1}$, vis-à-vis the formal $4 \mathrm{f}^{7}$.

Ground and Low-Lying Excited States. The molecular orbitals that are listed in Table 4 are used as the active space of the LFDFT calculation of Eu configuration $4 \mathrm{f}^{7}$ in the molecular $\mathrm{Eu}\left(\eta^{9}-\mathrm{C}_{9} \mathrm{H}_{9}\right)_{2}$ complex. The ligand-field parameters are derived from the listed orbitals, ${ }^{32,33}$ which include the Slater-Condon integrals $\left(F^{2}(4 \mathrm{f}, 4 \mathrm{f}), F^{4}(4 \mathrm{f}, 4 \mathrm{f})\right.$, and $\left.F^{6}(4 \mathrm{f}, 4 \mathrm{f})\right)$ and the ligand-field potential (a matrix with $7 \times 7$ elements). Besides, the spin-orbit coupling interaction is also incorporated by performing ZORA calculations at the spin-orbit level of theory, ${ }^{45}$ obtaining from DFT the spin-orbit coupling constant $\left(\zeta_{4 \mathrm{f}}\right)$. In the Supporting Information, Tables S3 and S4 list the calculated parameters that are obtained with DFT employing the LDA, PBE, and B3LYP functionals.
Similarly, the molecular orbitals listed in Table 5 are used as the active space of the LFDFT calculation of Eu configuration $4 \mathrm{f}^{6} 5 \mathrm{~d}^{1}$ in the molecular $\mathrm{Eu}\left(\eta^{9}-\mathrm{C}_{9} \mathrm{H}_{9}\right)_{2}$ complex. The ligandfield parameters are also extracted from the listed orbitals, ${ }^{32,33}$ which include the Slater-Condon integrals $\left(F^{2}(4 f, 4 f)\right.$, $F^{4}(4 f, 4 f), \quad F^{6}(4 f, 4 f), \quad G^{1}(4 f, 5 d), \quad G^{3}(4 f, 5 d), \quad G^{5}(4 f, 5 d)$, $\mathrm{F}^{2}(4 \mathrm{f}, 5 \mathrm{~d})$, and $\mathrm{F}^{4}(4 \mathrm{f}, 5 \mathrm{~d})$ ), the ligand-field potential (a matrix with $12 \times 12$ elements), and the energy gap $\left(\Delta E_{\mathrm{av}}\right)$. The spinorbit coupling is also calculated by estimating the constant $\left(\zeta_{4 \mathrm{f}}\right.$ and $\zeta_{5 \mathrm{~d}}$ ) from the ZORA spin-orbit calculation. ${ }^{45}$ In the Supporting Information, Tables S5 and S6 list the calculated parameters that are obtained with DFT employing the LDA, PBE, and B3LYP functionals.

However, it is noteworthy to mention here that we redefine the calculation of the energy gap $\left(\Delta E_{\mathrm{av}}\right)$ parameter. ${ }^{32,33}$ In LFDFT, which is implemented in the ADF suite of software, ${ }^{29-31} \Delta E_{\mathrm{av}}$ represents the difference between DFT total energies $\left(E^{\mathrm{DFT}}\right)$, working with the AOC fractional electron occupation, the molecular complex being at two different electron configuration states. In other words, we obtain $\Delta E_{\mathrm{av}}=E^{\mathrm{DFT}}\left(4 \mathrm{f}^{6} 5 \mathrm{~d}^{1}\right)-E^{\mathrm{DFT}}\left(4 \mathrm{f}^{7}\right)$. However, by this definition, the calculated $\Delta E_{\mathrm{av}}$ parameter is always overestimated, giving rise to shifting of the excitation energies to higher energy if compared to the experiments. This is not dramatic if we consider all of the recent application of LFDFT in relation to the $\mathrm{X}$-ray spectroscopy studies of coordination compounds. $^{60-63}$ However, for application like the present photoluminescence properties study, the shift of the excitation energies is relatively quite large. Therefore, we propose a new definition of the $\Delta E_{\text {av }}$ parameter, which we obtain directly in relation with the molecular orbital energies listed in Tables 4 and 5. Thus, we propose $\Delta E_{\mathrm{av}}=\bar{E}_{5 \mathrm{~d}}^{\mathrm{DFT}}\left(4 \mathrm{f}^{6} 5 \mathrm{~d}^{1}\right)-\bar{E}_{4 \mathrm{f}}^{\mathrm{DFT}}\left(4 \mathrm{f}^{7}\right)$, where $\bar{E}_{5 \mathrm{~d}}^{\mathrm{DFT}}\left(4 \mathrm{f}^{6} 5 \mathrm{~d}^{1}\right)$ and $\bar{E}_{4 \mathrm{f}}^{\mathrm{DFT}}\left(4 \mathrm{f}^{7}\right)$ represent the barycenters of the ligand-field splitting manifold of the $5 \mathrm{~d}$ and $4 \mathrm{f}$ orbitals obtained from DFT calculations of the molecular complex with Eu configurations $4 f^{6} 5 \mathrm{~d} 1$ and $4 \mathrm{f}^{7}$, respectively. In practice, if we employ the former definition of the parameter, we obtain $\Delta E_{\mathrm{av}}$ $=4.0692 \mathrm{eV}$ (DFT calculation with the PBE functional), whereas if we consider the new definition, $\Delta E_{\mathrm{av}}$ becomes $2.7517 \mathrm{eV}$, improving considerably the excitation energy values, enhancing ipso facto the agreement between theoretical and experimental spectral profiles (vide infra).

Figure 3 shows the calculated excitation energies, $\Delta E_{i}^{(0)}=$ $\left(E_{i}^{(0)}-E_{1}^{(0)}\right)$ and $\Delta E_{i}^{(1)}=\left(E_{i}^{(1)}-E_{1}^{(0)}\right)$, that result from the Eu $4 \mathrm{f}^{7} \rightarrow 4 \mathrm{f}^{7}$ (red bars) and $4 \mathrm{f}^{7} \rightarrow 4 \mathrm{f}^{6} 5 \mathrm{~d}^{1}$ (blue bars) electron transitions, respectively. It shows the energy levels that are located in the energy range between 0 and $3.5 \mathrm{eV}$, highlighting the calculated ground and low-lying excited states (ESs) of $\mathrm{Eu}\left(\eta^{9}-\mathrm{C}_{9} \mathrm{H}_{9}\right)_{2}$ obtained by means of LFDFT using the LDA, PBE, and B3LYP DFT functionals. There are in total 1716 and 15015 Kramers doublets that form the multiplet manifold of the Eu configurations $4 \mathrm{f}^{7}$ and $4 \mathrm{f}^{6} 7 \mathrm{~d}^{1}$, respectively.

The GS of $\mathrm{Eu}\left(\eta^{9}-\mathrm{C}_{9} \mathrm{H}_{9}\right)_{2}$ is derived from the ${ }^{8} \mathrm{~S}_{7 / 2}$ term of the $\mathrm{Eu}^{2+}$ configuration $4 \mathrm{f}^{7}$. This atomic term is split in energy into four Kramers doublets via zero-field splitting in the molecular $\mathrm{Eu}\left(\eta^{9}-\mathrm{C}_{9} \mathrm{H}_{9}\right)_{2}$ complex. These four Kramers doublets can be separately discriminated in terms of their calculated $M_{J}$ values, which are good quantum numbers in $D_{9 h}$ symmetry. Therefore, we obtain a state with $M_{J}= \pm 7 / 2$ as the zero of the energy, independent of the choice of DFT functional. We obtain also that, in order of increasing energy, the states with $M_{J}= \pm 5 / 2, \pm 3 / 2$, and $\pm 1 / 2$ are low-lying ESs 


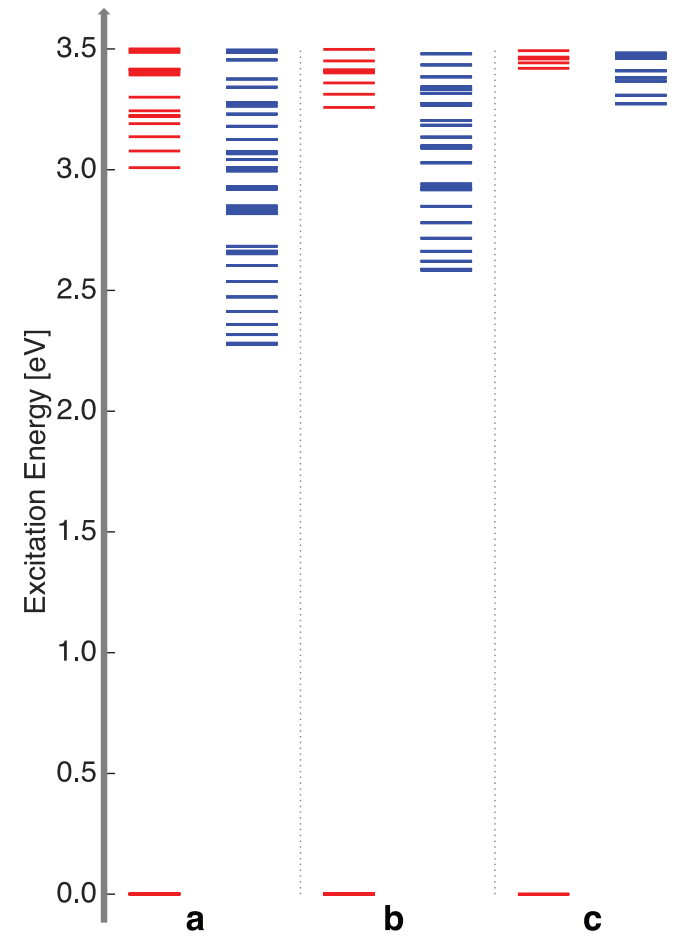

Figure 3. Ground and low-lying ESs of the molecular $\mathrm{Eu}\left(\eta^{9}-\mathrm{C}_{9} \mathrm{H}_{9}\right)_{2}$ complex obtained from LFDFT with LDA (a), PBE (b), and B3LYP (c) DFT functionals, showing the vertical excitation energies that result from Eu $4 \mathrm{f}^{7} \rightarrow 4 \mathrm{f}^{7}$ (red bars) and $\mathrm{Eu} 4 \mathrm{f}^{7} \rightarrow 4 \mathrm{f}^{6} 5 \mathrm{~d}^{1}$ transitions (blue bars). with very small energy separations, vis-à-vis, the GS (tens of $\mathrm{meV})$.

The LFDFT calculations indicate that the multiplet manifolds of the $\mathrm{Eu}^{2+}$ configurations $4 \mathrm{f} 7$ and $4 \mathrm{f}^{6} 7 \mathrm{~d}^{1}$ overlap in terms of energy (see Figure 3). Most significant, some energy levels corresponding to $4 \mathrm{f}^{6} 7 \mathrm{~d}^{1}$ are predicted below the next levels of the $4 \mathrm{f}^{7}$, independent of the choice of DFT functional. The LDA and PBE results allow identification of relatively many ESs in this situation. We note that, in general, preferred $\mathrm{Eu}^{2+}$ materials are tailored to exhibit low-lying $4 \mathrm{f}^{6} 7 \mathrm{~d}^{1}$ ESs. ${ }^{64}$ The excitation to and emission from these states are fundamental in the mechanism of the visible light generation induced by the materials. These ESs exhibit a strong admixture of $\mathrm{Eu}^{2+}$ atomic spectral terms, in particular, due to the large ligand-field splitting of the Eu $5 \mathrm{~d}$ orbitals. Thus, the lowest $4 \mathrm{f}^{6} 7 \mathrm{~d}^{1}$ Kramer doublets in Figure 3 are characterized by admixture of the ${ }^{8,6} \mathrm{P},{ }^{8,6} \mathrm{D},{ }^{8,6} \mathrm{~F},{ }^{8,6} \mathrm{G}$, and ${ }^{8,6} \mathrm{H}$ atomic spectral terms of configuration $4 f^{6} 7 d^{1}$ resulting from the $4 f^{6}\left({ }^{7} \mathrm{~F}\right) \otimes 5 \mathrm{~d}^{1}$ $\left({ }^{2} \mathrm{D}\right)$ direct product.

Photoluminescence-Excitation Spectrum. Figure 4a shows the calculated excitation spectra of the molecular $\operatorname{Eu}\left(\eta^{9}\right.$ $\left.\mathrm{C}_{9} \mathrm{H}_{9}\right)_{2}$ complex, obtained from LFDFT on the basis of the Eu $4 \mathrm{f}^{7} \rightarrow 4 \mathrm{f}^{6} 7 \mathrm{~d}^{1}$ electron transitions. The spectra are obtained by computing the oscillator strengths of the electric-dipoleallowed transitions, $I_{i} \cong\left|\left\langle\psi_{i}^{(1)}|d| \psi_{1}^{(0)}\right\rangle\right|^{2}$ as a function of the excitation energies, $\Delta E_{i}^{(1)}=\left(E_{i}^{(1)}-E_{1}^{(0)}\right)$, with $i=1,2,3, \ldots$, 30030, (bars in Figure 4a). The intensity involves the transition moment function $\left\langle\psi_{i}^{(1)}|d| \psi_{1}^{(0)}\right\rangle$ and the electricdipole moment operator $d$. In the transition moment function,
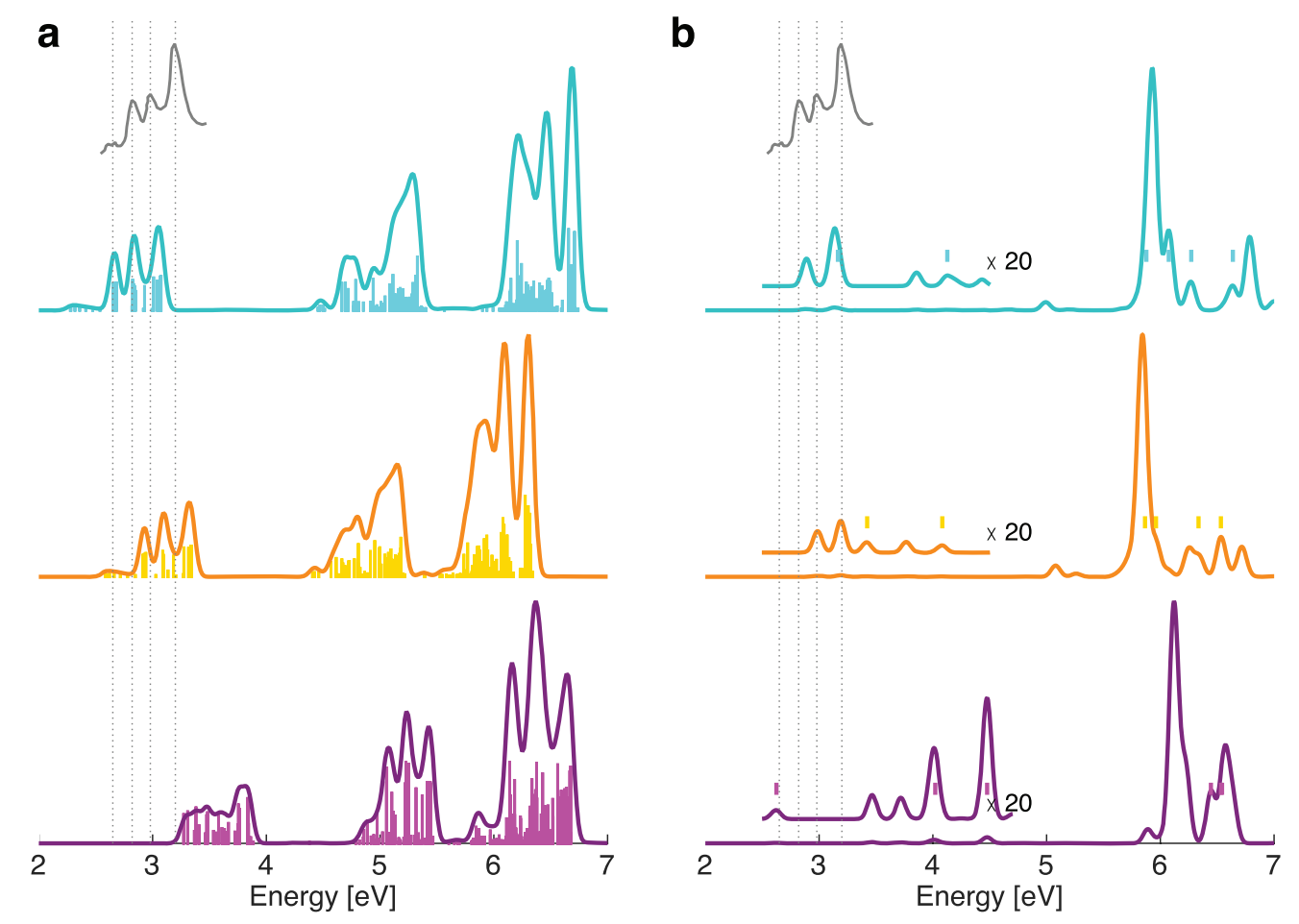

Figure 4. Graphical representations of the calculated excitation spectra of the molecular $\mathrm{Eu}\left(\eta^{9}-\mathrm{C}_{9} \mathrm{H}_{9}\right)_{2}$ complex (in arbitrary unit) as a function of the photon energy (in eV), obtained from LFDFT (a) and TDDFT (b) methods using the LDA (top), PBE (middle), and B3LYP (bottom) DFT functionals. The excitation spectra result from the broadening of the calculated oscillator strengths with a Gaussian function with a constant halfwidth at half-maximum parameter $(0.040 \mathrm{eV})$. In the LFDFT results section, the transition probabilities $\left\langle\left|\psi_{i}^{(1)}\right| d\left|\psi_{1}^{(0)}\right|\right\rangle^{2}$ are shown $(\mathrm{cyan}$, orange, and magenta bars) in arbitrary unit versus the excitation energies. In the TDDFT results section, the energy occurrences of the predominant intraatomic Eu transitions are also shown (cyan, orange, and magenta bars). Experimental data that has been previously reported in ref 20 is reproduced (gray curves) for comparison. 
the initial state $\left(\psi_{1}^{(0)}\right)$ represents the 8 -fold quasi-degenerate electronic states of $\mathrm{Eu}\left(\eta^{9}-\mathrm{C}_{9} \mathrm{H}_{9}\right)_{2}$ derived from the ${ }^{8} \mathrm{~S}_{7 / 2}$ term of configuration $4 \mathrm{f}^{7}$ (see also Figure 3) without considering the temperature effect. The final states $\left(\psi_{i}^{(1)}\right.$, with $i=1,2,3, \ldots$, 30030) form the basis of the whole manifold of the ESs of $\mathrm{Eu}\left(\eta^{9}-\mathrm{C}_{9} \mathrm{H}_{9}\right)_{2}$ that arise from $\mathrm{Eu}^{2+}$ configuration $4 \mathrm{f}^{6} 5 \mathrm{~d}^{1}$ (see also Figure 3).

We use a Gaussian function to broaden the calculated oscillator strengths $\left(I_{i}\right)$ in Figure 4. The Gaussian function approximates with a constant half-width at half-maximum parameter the finite experimental energy resolution, generating a theoretical spectral profile close to the experimental findings. ${ }^{65}$ In Figure 4a, the LFDFT results that are obtained with the LDA, PBE, and B3LYP DFT functionals are represented. The experimental excitation spectrum of $\mathrm{Eu}\left(\eta^{9}\right.$ $\left.\mathrm{C}_{9} \mathrm{H}_{9}\right)_{2}$ that is taken from ref 20 is also reproduced, for direct comparison with the theoretical results.

The calculated excitation spectra are characterized by three main absorption bands because of the large ligand-field splitting of the 5d orbitals (see Figure 4). The three absorption bands are well separated in energy. That is, in line with the ligand-field splitting of the Eu 5d orbitals, which follows the sequence $\mathrm{a}_{1}{ }^{\prime}\left(5 \mathrm{~d}_{\sigma}\right)<\mathrm{e}_{2}{ }^{\prime}\left(5 \mathrm{~d}_{\delta}\right)<\mathrm{e}_{1}{ }^{\prime \prime}\left(5 \mathrm{~d}_{\pi}\right)$, we find that the first band at circa $3 \mathrm{eV}$ represents the $\mathrm{Eu} 4 \mathrm{f} \rightarrow 5 \mathrm{~d}_{\sigma}$ transitions. Similarly, the second band at circa $5 \mathrm{eV}$ represents the Eu $4 \mathrm{f} \rightarrow$ $5 \mathrm{~d}_{\delta}$ transitions. Finally, the third band at circa $6 \mathrm{eV}$ consists of the $\mathrm{Eu} 4 \mathrm{f} \rightarrow 5 \mathrm{~d}_{\pi}$ transitions. We also observe that on top of the absorption bands fine structures are also present (see Figure 4a) due to the interelectron repulsion and spin-orbit coupling terms of the model Hamiltonian.

In the experiment (the excitation spectrum was recorded at $77 \mathrm{~K}){ }^{20}$ we observe that only a limited range of the whole spectrum was recorded. This mainly corresponds to the Eu $4 \mathrm{f}$ $\rightarrow 5 \mathrm{~d}_{\sigma}$ transitions, where the absorption band is characterized by three peaks at energies of $2.82 \mathrm{eV}, 2.98$, and $3.20 \mathrm{eV}$, as well as one small shoulder at an energy of $2.65 \mathrm{eV}$ (see Figure 4). Our theoretical results reveal some concrete resemblance to the experimental data.

Namely, the theoretical spectral profiles exhibit the same three peaks and one shoulder representative of the experimental excitation spectrum. In the LDA and PBE results, the three peaks and one shoulder are remarkably well resolved, which are in contrast to the B3LYP results (see Figure 4a). In addition, we note some energy shift in the calculated excitation energies, if compared to the experiment. Overall, the PBE results offer the best theoretical reproduction of the experiment in terms of spectral profile and excitation energies. The LDA and B3LYP results slightly deviate from the experiment, obtaining underestimated and overestimated excitation energies, respectively.

To complement the results obtained from the LFDFT method, we also report in Figure $4 \mathrm{~b}$ the results of the TDDFT calculations of the excitation energies and optical properties of the molecular $\mathrm{Eu}\left(\eta^{9}-\mathrm{C}_{9} \mathrm{H}_{9}\right)_{2}$ complex. The excitation energies and eigenfunctions of the ESs are determined from unrestricted TDDFT by using the ADF suite of software. ${ }^{29-31}$ We employ the LDA and PBE functional with the asymptotically correct statistical average of orbitals model potential (SAOP), ${ }^{66,67}$ as well as the B3LYP functional. By using the TDDFT method, we aim at obtaining the excitation energies that correspond to the $4 \mathrm{f} \rightarrow 5 \mathrm{~d}$ transitions as well as some charge transfer transitions of ligand-to-metal $(\pi \rightarrow 4 \mathrm{f}, 5 \mathrm{~d})$, metal-to-ligand $\left(4 \mathrm{f}, 5 \mathrm{~d} \rightarrow \pi^{*}\right)$, and ligand-to-ligand $\left(\pi \rightarrow \pi^{*}\right)$ kinds. Thus, we aspire to establish a complete understanding of the photoluminescence properties of $\mathrm{Eu}\left(\eta^{9}-\mathrm{C}_{9} \mathrm{H}_{9}\right)_{2}$ on the basis of all possible electron transitions that are resolved by both LFDFT and TDDFT methods. Note, however, that we are limited in the TDDFT calculations to the scalar relativistic level of theory. In other words, in Figure $4 b$, no spin-orbit coupling interaction is taken into consideration.

The spectroscopic trends are quasi-identical for the three DFT functionals under consideration (see Figure 4b). Thus, we restrict our discussion of the TDDFT results that are obtained at the PBE level. The molecular $\mathrm{Eu}\left(\eta^{9}-\mathrm{C}_{9} \mathrm{H}_{9}\right)_{2}$ complex possesses strongly allowed transitions at $5.8441 \mathrm{eV}$ onward (as results of the PBE calculation but similarly at 5.9369 and $6.1195 \mathrm{eV}$ for the LDA and B3LYP results, respectively), which are interpreted as an admixture of ligandto-ligand $\left(\pi \rightarrow \pi^{*}\right)$ charge transfer and Eu $4 \mathrm{f} \rightarrow 5 \mathrm{~d}$ transitions. Considering the intra-atomic $\mathrm{Eu}$ ff $\rightarrow 5 \mathrm{~d}$ obtained with nonzero transition probabilities, we identify transitions with predominant $\mathrm{Eu}\left(4 \mathrm{f}_{\sigma} \rightarrow 5 \mathrm{~d}_{\sigma}\right)$ at 3.4221 and $4.0829 \mathrm{eV}$. The transitions occur at two different energies due to the mixing between Eu $5 \mathrm{~d}$ and $6 s$ orbitals, which possess the same $\mathrm{a}_{1}{ }^{\prime}$ representation in $D_{9 h}$ symmetry (see also Figure 2). Besides, the calculated oscillator strengths are relatively weak in the magnitude of $10^{-3}$ au. We also identify transitions with predominant $\mathrm{Eu}\left(4 \mathrm{f}_{\pi} \rightarrow 5 \mathrm{~d}_{\delta}\right)$ and $\mathrm{Eu}\left(4 \mathrm{f}_{\varphi} \rightarrow 5 \mathrm{~d}_{\delta}\right)$ at 5.8651 and $5.9616 \mathrm{eV}$, respectively. These transitions are accompanied by relatively stronger intensity in the magnitude of $10^{-1}$ au. Other transitions having similar intensity magnitude are found at 6.3360 and $6.5323 \mathrm{eV}$, corresponding predominantly to the $\mathrm{Eu}\left(4 \mathrm{f}_{\delta} \rightarrow 5 \mathrm{~d}_{\pi}\right)$ and $\mathrm{Eu}\left(4 \mathrm{f}_{\sigma} \rightarrow 5 \mathrm{~d}_{\pi}\right)$, respectively. In Figure $4 \mathrm{~b}$, the energy occurrences of the aforementioned electronic transitions are individually highlighted. Below $5.8441 \mathrm{eV}$, we also observe transitions with weaker intensities. These transitions principally originate from metal-to-ligand charge transfer. For instance, we observe the two small bumps at 2.9863 and $3.1901 \mathrm{eV}$, which represent the $\left(4 \mathrm{f}_{\delta} \rightarrow \pi^{*} \mathrm{e}_{3}{ }^{\prime \prime}\right)$ and $\left(4 \mathrm{f}_{\varphi} \rightarrow \pi^{*} \mathrm{e}_{3}{ }^{\prime \prime}\right)$ transitions, respectively.

In LFDFT, multiplet effects are rigorously treated, resulting from configuration interaction within Eu configuration $4 f^{6} 5 d^{1}$. Therefore, a direct comparison between the excitation energies obtained from LFDFT (Figure 4a) and TDDFT (Figure 4b) is not practicable. However, in general, the calculated excitation energies corresponding to the $\mathrm{Eu} 4 \mathrm{f} \rightarrow 5 \mathrm{~d}$ transitions that we obtain from TDDFT and LFDFT are found to be in good agreement. We must point out that it is possible that the experimental spectral profile combines both intra-atomic Eu $4 \mathrm{f}$ $\rightarrow 5 \mathrm{~d}$ and metal-to-ligand charge transfer transitions. Because LFDFT is restricted only to the study of the intra-atomic Eu transitions, in our perspective, the TDDFT results bring complementary insight in relation to the possible other transitions that also play a role in the full photoluminescence properties of $\mathrm{Eu}\left(\eta^{9}-\mathrm{C}_{9} \mathrm{H}_{9}\right)_{2}$.

Photoluminescence-Emission Spectrum. In this section, we present a rather simplified account for calculation of the emission spectra corresponding to the $\mathrm{Eu} 5 \mathrm{~d} \rightarrow 4 \mathrm{f}$ transitions. We do not address here a rigorous analysis of the relaxation process on the basis of many ESs and many possible paths with nonradiative decay (Kasha's rule). ${ }^{68}$ As a simple assumption, we consider the $5 \mathrm{~d} \rightarrow 4 \mathrm{f}$ emission as the result of radiation from the calculated lowest multiplet state corresponding to the Eu configuration $4 \mathrm{f}^{6} 5 \mathrm{~d}^{1}$ to the GS of the Eu configuration $4 \mathrm{f}^{7}$. In the other words, using the representation in Figure 3, we idealize the emission from the lowest blue level 
(initial state) to the GS red level (final state) giving rise to a single zero-phonon line, which is further subjected to vibronic coupling. ${ }^{35-37}$

We have to consider prior investigation of the emission radiation itself, a structural relaxation of the molecular $\operatorname{Eu}\left(\eta^{9}\right.$ $\left.\mathrm{C}_{9} \mathrm{H}_{9}\right)_{2}$ complex in the ES with Eu configuration $4 \mathrm{f}^{6} 5 \mathrm{~d}^{1}$ as well as LFDFT calculations of the multiplet energies of both Eu $4 \mathrm{f}^{7}$ and $4 \mathrm{f}^{6} 5 \mathrm{~d}^{1}$ at this excited geometry. We optimize again the geometry of the molecular $\mathrm{Eu}\left(\eta^{9}-\mathrm{C}_{9} \mathrm{H}_{9}\right)_{2}$ complex with a constraint on the electronic structure. We use the same procedure as that described in the Methods section, apart that we now target geometries at the excited $\mathrm{Eu}$ configuration $4 f^{6} 5 d^{1}$. The open-shell $4 f$ electrons are treated with fractional orbital occupations, i.e., the seven molecular orbitals with predominant $\mathrm{Eu} 4 \mathrm{f}$ character are occupied with $6 / 7$ electrons. This is a rather technical aspect that helps us ensure the SCF convergence, while the strongly correlated nature of the $4 \mathrm{f}$ electrons cannot be represented by a single determinant. ${ }^{52,53}$ The open-shell $5 \mathrm{~d}$ electrons, on the other hand, are treated by placing one electron in the molecular orbital with $\mathrm{a}_{1}{ }^{\prime}$ representation in $D_{9 h}$ that has predominantly $5 \mathrm{~d}_{\sigma}$ character. We relax the structure conserving the initial $D_{9 h}$ symmetry. We note that the selective occupation of the $a_{1}{ }^{\prime}$ orbital with predominant $\mathrm{Eu} 5 \mathrm{~d}_{\sigma}$ helps maintain the symmetry, being a nondegenerate state ruling out possible distortion of the atomic structure due to the Jahn-Teller effect. ${ }^{35-37}$

Table 6 lists the calculated geometries of $\mathrm{Eu}\left(\eta^{9}-\mathrm{C}_{9} \mathrm{H}_{9}\right)_{2}$ at the excited $\mathrm{Eu}$ configuration $4 \mathrm{f}^{6} 5 \mathrm{~d}^{1}$ obtained from DFT

Table 6. Calculated Geometrical Parameters for $\mathrm{Eu}\left(\boldsymbol{\eta}^{9}\right.$ $\left.\mathrm{C}_{9} \mathrm{H}_{9}\right)_{2}$ in the Excited Eu Configuration $4 \mathrm{f}^{6} 5 \mathrm{~d}^{1}$ (ES) within the $D_{9 h}$ Point Group: Bond Distances $(d$ in $\AA$ ) and Bond Angles $(A$ in deg)

$\begin{array}{cccc}D_{9 h} & \text { LDA } & \text { PBE } & \text { B3LYP } \\ d(\mathrm{Eu}-\mathrm{C}) & 2.729 & 2.811 & 2.899 \\ d(\mathrm{C}-\mathrm{X})^{a} & 2.038 & 2.056 & 2.047 \\ d(\mathrm{C}-\mathrm{H}) & 1.100 & 1.097 & 1.086 \\ d(\mathrm{Eu}-\mathrm{X})^{a} & 1.815 & 1.917 & 2.053 \\ A(\mathrm{X}-\mathrm{C}-\mathrm{H})^{a} & 175.2 & 175.9 & 176.3\end{array}$

${ }^{a} \mathrm{X}$ represents the centroid of the $\left(\eta^{9}-\mathrm{C}_{9} \mathrm{H}_{9}\right)^{-}$ring.

calculations using the LDA, PBE, and B3LYP functionals. The atomic structures of $\mathrm{Eu}\left(\eta^{9}-\mathrm{C}_{9} \mathrm{H}_{9}\right)_{2}$ vary from Eu configuration $4 \mathrm{f}^{7}$ (Table 2) to $4 \mathrm{f}^{6} 5 \mathrm{~d}^{1}$ (Table 6). More particularly, the $4 \mathrm{f} \rightarrow$ $5 \mathrm{~d}$ electron excitation leads to a shortening of the $\mathrm{Eu}-\mathrm{C}$ bond distances, which signifies that the occupation of the $5 \mathrm{~d}$ orbitals strengthens the chemical bonding between Eu and the ligands. This $\mathrm{Eu}-\mathrm{C}$ bond length variation results in a small vibrational frequency, displayed as ring approchement and removal vibration at 48 and $47 \mathrm{~cm}^{-1}$ for $D_{9 d}$ and $D_{9 h}$ symmetries, respectively, with an out-of-plane ring tilting.

The shortening of the $\mathrm{Eu}-\mathrm{C}$ bond distance is comparable to that from earlier studies of lanthanide complexes, ${ }^{42-44,57}$ showing that $4 \mathrm{f}$ contributes weakly to the chemical bonding, where bonding is typically ionic and partly due to donation effects into the empty $5 \mathrm{~d}$. The change in the bond distance is significant, by about $2 \%$ responsible in part of the observation of the Stokes shift ${ }^{69}$ in the excitation-emission spectra.

The Eu $5 \mathrm{~d} \rightarrow 4 \mathrm{f}$ emission line is coupled to vibrations because of different GS and ES geometries of $\mathrm{Eu}\left(\eta^{9}-\mathrm{C}_{9} \mathrm{H}_{9}\right)_{2}$. We neglect the thermal occupation of the vibrational ESs in the electronic $4 \mathrm{f}^{6} 5 \mathrm{~d}^{1}$ states. That is, at a temperature of $0 \mathrm{~K}$, the transitions take place only from the vibrational quantum number $n=0$ of the lowest electronic $4 \mathrm{f}^{6} 5 \mathrm{~d}^{1}$ state to the manifold of the vibrational quantum number $n$ of the electronic $4 \mathrm{f}^{7}$ GS. The strengths of these transitions are a priori modeled through the Franck-Condon factors. ${ }^{70}$

The Franck-Condon factors are obtained by using the twodimensional array method in ref 70 that requires the calculation of the harmonic frequencies and normal modes at the two stationary GS and ES points of $\mathrm{Eu}\left(\eta^{9}-\mathrm{C}_{9} \mathrm{H}_{9}\right)_{2}$. The correlations between the normal modes of the GS and ES are constructed by means of the orthonormal Dushinsky matrix. ${ }^{71}$ Practically, the displacement vector $R$ that is calculated such that $R=R_{\mathrm{ES}}-R_{\mathrm{GS}}$ is expressed as ${ }^{72}$

$$
R=\sum_{k} w_{k} Q_{k}^{(\mathrm{GS})}
$$

where $w_{k}$ represents the contribution of the displacement along the mass-weighted GS normal coordinates $\left(Q_{k}^{(\mathrm{GS})}\right)$ with $k=1$, $2, \ldots, 105$ (as per the total number of available vibrational modes $)$. The molecular $\left.\mathrm{Eu}\left(\eta^{9}-\mathrm{C}_{9} \mathrm{H}_{9}\right)_{2}\right)$ complex has $3 \mathrm{~N}-6=$ 105 vibrational modes (where $N$ stands for the total number of atoms in the molecule, i.e., 37), which are classified according to the representations of the $D_{9 h}$ point group: $4 \mathrm{a}_{1}{ }^{\prime}+1 \mathrm{a}_{2}{ }^{\prime}+6$ $\mathrm{e}_{1}{ }^{\prime}+6 \mathrm{e}_{2}{ }^{\prime}+6 \mathrm{e}_{3}{ }^{\prime}+6 \mathrm{e}_{4}{ }^{\prime}+2 \mathrm{a}_{1}{ }^{\prime \prime}+4 \mathrm{a}_{2}{ }^{\prime \prime}+5 \mathrm{e}_{1}{ }^{\prime \prime}+6 \mathrm{e}_{2}{ }^{\prime \prime}+6 \mathrm{e}_{3}{ }^{\prime \prime}$ $+6 \mathrm{e}_{4}^{\prime \prime}$. Table 7 lists the calculated displacement vector $w_{k}$ with respect to the DFT functional.

Table 7. Analysis of the Changes in Geometry of $\mathrm{Eu}\left(\eta^{9}\right.$. $\left.\mathrm{C}_{9} \mathrm{H}_{9}\right)_{2}$ from Eu $4 f^{7}$ to $4 f^{6} 5 d^{1}$ (Obtained from DFT Using the LDA, PBE, and B3LYP Functionals), Listing the Displacement Vector $w_{k}\left(\right.$ in $\left.\mathrm{amu}^{1 / 2} \cdot \AA\right)$ and Huang-Rhys Factor $S_{k}([-])$ as a Function of the Totally Symmetric Vibrational Modes $\left(\mathrm{a}_{1}{ }^{\prime}\right)$ with Frequencies $\hbar \omega\left(\right.$ in $\left.\mathrm{cm}^{-1}\right)$ Obtained in the GS and ES

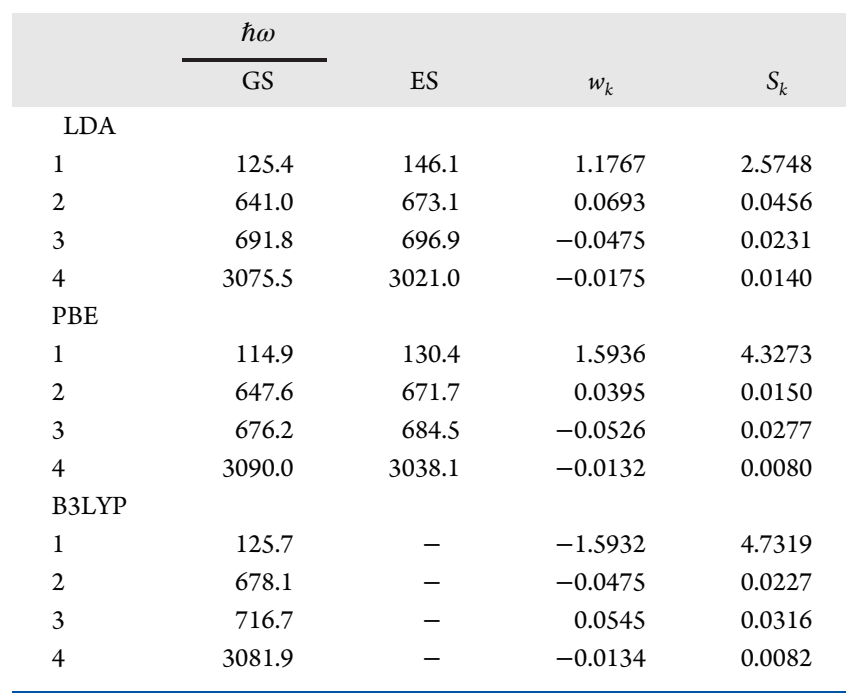

Because the $4 \mathrm{f} \rightarrow 5 \mathrm{~d}$ excitation process conserves the $D_{9 h}$ symmetry, only the four totally symmetric vibrations $\left(\mathrm{a}_{1}{ }^{\prime}\right)$ are involved in the displacement vector and therefore play a role in the emission spectra. The graphical representations of these vibrational modes are shown in the Supporting Information Figure S2. The dimensionless Huang-Rhys factors $\left(S_{k}\right)$ that represent the strength of the linear vibronic coupling between the electronic state and the $\mathrm{a}_{1}{ }^{\prime}$ modes are calculated as a function of $w_{k}$ and the reduced frequencies at the GS 
structure. ${ }^{73}$ In Table 7 , we list also the frequencies $(\hbar \omega)$ of the $\mathrm{a}_{1}{ }^{\prime}$ modes obtained at the GS and ES, together with the calculated $S_{k}$. The differences between the frequencies of the $\mathrm{a}_{1}{ }^{\prime}$ modes at the GS and ES atomic structures are relatively small as results of the LDA and B3LYP calculations. For the B3LYP calculation, it was not possible to access frequencies at ESs. Therefore, we compromise to use the same vibrational wave functions for both GS and ES.

The Franck-Condon factors are calculated as follows, ${ }^{74}$ $F_{k}(0, n)=\frac{\mathrm{e}^{-S_{k}} S_{k}^{n}}{n !}$, with $k=1, \ldots, 4$ for the four $\mathrm{a}_{1}{ }^{\prime}$ modes involved in the displacement vector as a function of the calculated Huang-Rhys factor $\left(S_{k}\right)$ and the GS vibrational state quantum number $n$. The 0 notation in the expression of the Franck-Condon factor means that only the $n=0$ ES vibrational state quantum number is taken into consideration. The energy occurrences of the Franck-Condon factors are obtained as follows

$$
\begin{aligned}
E_{k}(0, n)= & \left(E_{1}^{(1)}-E_{1}^{(0)}\right)-\left(\left(n+\frac{1}{2}\right) \hbar \omega_{\mathrm{GS}, k}\right. \\
& \left.-\left(0+\frac{1}{2}\right) \hbar \omega_{\mathrm{ES}, k}\right)
\end{aligned}
$$

where $E_{1}^{(1)}$ and $E_{1}^{(0)}$ are the lowest energy of the $4 \mathrm{f}^{6} 5 \mathrm{~d}^{1}$ multiplet manifold and the GS energy of the $4 \mathrm{f}^{7}$, respectively, that are obtained from LFDFT calculations of the ES structure.

Figure 5 shows the calculated emission spectra of the molecular $\mathrm{Eu}\left(\eta^{9}-\mathrm{C}_{9} \mathrm{H}_{9}\right)_{2}$ complex compared with the experiment (the experimental emission spectrum was recorded at 77 $\mathrm{K}){ }^{20}$ Multiple transitions occur in the emission process, giving rise to a spectral profile with a slightly antisymmetric Gaussian shape independent of the DFT functional, which is in agreement with the experimental data. ${ }^{20}$ The four $a_{1}{ }^{\prime}$ vibrational modes (see the Supporting Information Figure S1) are responsible for the spectral profile, the associated Franck-Condon factors being represented by the blue, red, green, and black bars in Figure 5. The first $a_{1}{ }^{\prime}$ mode, $a$ symmetric breathing vibration, has significant contribution to the antisymmetry of the Gaussian profile of the emission (see the blue bars in Figure 5), having a relatively important Huang-Rhys factor independent of the choice of the DFT functional (see Table 7). The other $\mathrm{a}_{1}{ }^{\prime}$ modes are less critical (for the apparent reason that their associated Franck-Condon factors almost merge in Figure 5) because they are associated with a relatively small Huang-Rhys factor (see Table 7). Overall, the PBE results are the closest to the experimental data (Figure 5), predicting an emission line at $2.434 \mathrm{eV}$ (i.e., $510 \mathrm{~nm}$ ) in the blue-green visible light spectral region with good agreement with the experiment. ${ }^{20}$

\section{CONCLUSIONS}

We studied the electronic structure and photoluminescence properties of the molecular $\mathrm{Eu}\left(\eta^{9}-\mathrm{C}_{9} \mathrm{H}_{9}\right)_{2}$ complex on the basis of the $\mathrm{Eu} 4 \mathrm{f}-5 \mathrm{~d}$ transitions. $\mathrm{Eu}^{2+}$ compounds have nowadays a critical impact on the development of future lighting technologies, with many applications in nanomaterials, bioimaging, biosensor, photocatalysis, etc. Therefore, an accurate method to calculate their electronic structure and to predict their optical properties is very relevant. We employed our LFDFT method to tackle the present problem with less computational effort and cost.

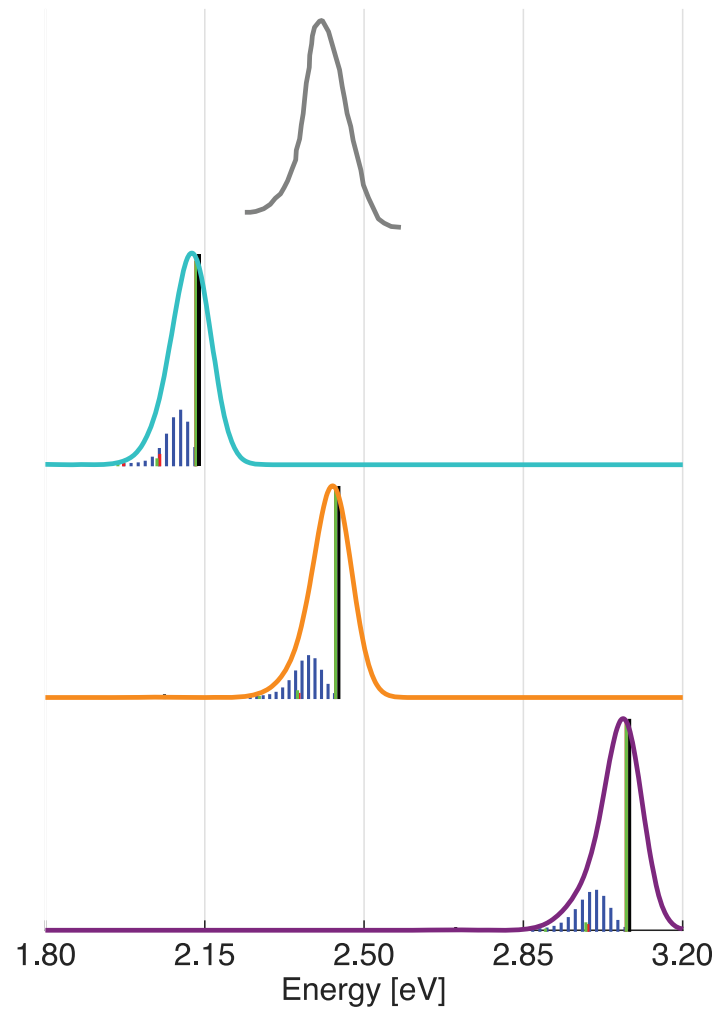

Figure 5. Graphical representations of the calculated emission spectra of $\mathrm{Eu}\left(\eta^{9}-\mathrm{C}_{9} \mathrm{H}_{9}\right)_{2}$ (in arbitrary units) as a function of the photon energy (in eV), obtained from the LFDFT method using the LDA (cyan curve), PBE (orange curve), and B3LYP (magenta curve) DFT functional. The emission spectra result from the broadening of the calculated Franck-Condon factors $F_{k}(0, n)$, with $k=1$ (blue bars), 2 (red bars), 3 (green bars), and 4 (black bars), for the four $\mathrm{a}_{1}{ }^{\prime}$ modes, with a Gaussian function with a constant half-width at half-maximum parameter $(0.040 \mathrm{eV})$. Experimental data that has been previously reported in ref 20 is reproduced (gray curve) for comparison.

In this work, we have determined the atomic structure of the molecular $\mathrm{Eu}\left(\eta^{9}-\mathrm{C}_{9} \mathrm{H}_{9}\right)_{2}$ complex by geometry optimization at the DFT level, giving rise to quasi-degenerate conformations with $D_{9 h}$ and $D_{9 d}$ symmetry (see Table 1$)$. We have obtained the atomic structures with a slight deviation vis-à-vis the experimental data (see Table 2) at the LDA, generalized gradient approximation (PBE), and hybrid (B3LYP) DFT functionals. Moreover, we have calculated the molecular orbitals diagram of $\mathrm{Eu}\left(\eta^{9}-\mathrm{C}_{9} \mathrm{H}_{9}\right)_{2}$, showing that the $\mathrm{Eu} 4 \mathrm{f}$ orbitals contribute only weakly to the chemical bonding, where bonding is typically ionic and partly due to donation effects into the virtual $\mathrm{Eu} 5 \mathrm{~d}$ orbitals, independent of the choice of DFT functional. The results of the calculation of the GS and low-lying ESs indicated that the excitation energies of the Eu $4 \mathrm{f}^{7} \rightarrow 4 \mathrm{f}^{7}$ and Eu $4 \mathrm{f}^{7} \rightarrow 4 \mathrm{f}^{6} 5 \mathrm{~d}^{1}$ overlapped in $\mathrm{Eu}\left(\eta^{9}-\mathrm{C}_{9} \mathrm{H}_{9}\right)_{2}$ (see Figure 3). The lowest calculated multiplet states of $4 \mathrm{f}^{6} 5 \mathrm{~d}^{1}$ are found below the excited multiplet states of $4 \mathrm{f}^{7}$, making $\mathrm{Eu}\left(\eta^{9}-\mathrm{C}_{9} \mathrm{H}_{9}\right)_{2}$ a good candidate for lighting material, taking advantage of strong optical effects due to the electric-dipoleallowed $4 \mathrm{f}-5 \mathrm{~d}$ transitions. We have simulated the excitation and emission spectra of $\mathrm{Eu}\left(\eta^{9}-\mathrm{C}_{9} \mathrm{H}_{9}\right)_{2}$ by LFDFT on the basis of the $4 \mathrm{f}^{7}\left({ }^{8} \mathrm{~S}\right) \rightarrow 4 \mathrm{f}^{6} 5 \mathrm{~d}^{1}$ and $4 \mathrm{f}^{6} 5 \mathrm{~d}^{1} \rightarrow 4 \mathrm{f}^{7}\left({ }^{8} \mathrm{~S}\right)$ transitions, respectively. The results showed that multiple transitions occurred at the excitation processes that are particularly governed by the two-electron repulsion and 5d ligand-field splitting terms of the model Hamiltonian (see Figure 4), giving 
rise to spectral profiles with multiple absorption bands and fine structures. The results also showed that the emission process has a strong vibronic origin (see Figure 5), which implied four totally symmetric vibrational modes on top of the electronic wave function, leading to a spectral profile with a slight antisymmetric Gaussian shape. The simulated excitation and emission spectra are found to be in total agreement with the experiments, in particular, for the PBE results.

The present work is aimed at presenting the performance of LFDFT for rather intricate problems in relation to the electronic structure and electron transition process. We provided an example for the application and performed comparative analysis with available experimental data and a time-dependent DFT tool. LFDFT is limited to the study of specific electron configuration systems, computing the multiplet energy levels and multielectronic eigenfunction as a function of single determinants of spin-orbitals. The LFDFT calculation is completely parameter-free and does not involve scaling factors and empirical corrections, enabling us to develop a fully theoretical model with a high prediction potential. Future studies include deeper investigation of lanthanides in the wide range of organometallic materials, in general, for a better understanding of the electronic structure and, in particular, for good knowledge of the microscopic origin of photoluminescence properties.

\section{ASSOCIATED CONTENT}

\section{S Supporting Information}

The Supporting Information is available free of charge at https://pubs.acs.org/doi/10.1021/acs.jpca.9b09755.

Geometrical parameters for $\mathrm{Eu}\left(\eta^{9}-\mathrm{C}_{9} \mathrm{H}_{9}\right)_{2}$ in the ground $\mathrm{Eu}$ configuration $4 \mathrm{f}^{7}$ as a function of a different numerical integration grid available in $\mathrm{ADF}$; graphical representations of the vibrations with the lowest frequencies in $\mathrm{Eu}\left(\eta^{9}-\mathrm{C}_{9} \mathrm{H}_{9}\right)_{2}$; calculated Slater-Condon integrals and spin-orbit coupling constants for $\mathrm{Eu}$ configuration $4 \mathrm{f}^{7}$; matrix element of the $4 \mathrm{f}$ ligand-field potential; calculated Slater-Condon integrals and spinorbit coupling constants for $\mathrm{Eu}$ configuration $4 \mathrm{f}^{6} 5 \mathrm{~d}^{1}$; matrix element of the merged $4 \mathrm{f}$ and $5 \mathrm{~d}$ ligand-field potential; and graphical representations of the four totally symmetric modes involved in the emission spectra through vibronic coupling (PDF)

\section{AUTHOR INFORMATION}

\section{Corresponding Authors}

*E-mail: harry.ra@hotmail.com.

*E-mail: henry.chermette@univ-lyon1.fr.

*E-mail: claude.daul@unifr.ch.

\section{ORCID (1)}

Harry Ramanantoanina: 0000-0002-2086-6958

Jules Tshishimbi Muya: 0000-0001-6761-9295

Henry Chermette: 0000-0002-5890-7479

\section{Author Contributions}

H.R. initiated the project. H.R and H.C. drafted the manuscript. All of the coauthors participated in the calculation including LFDFT, DFT, TDDFT, and group theory and contributed to revise the manuscript.

\section{Notes}

The authors declare no competing financial interest.

\section{ACKNOWLEDGMENTS}

The authors gratefully acknowledge the GENCI/CINES (Project cpt2130) and the university of Fribourg BEO04 for HPC resources/computer time. The authors thank Erwan Jeanneau for helpful discussions in the analysis of the crystal structure. H.C. and C. D. are glad to contribute to this issue in honor of Paul Geerlings, a great promoter of conceptual DFT.

\section{REFERENCES}

(1) Long, J.; Guari, Y.; Ferreira, R. A. S.; Carlos, L. D.; Larionova, J. Recent advances in luminescent lanthanide based single-molecule magnets. Coord. Chem. Rev. 2018, 363, 57-70.

(2) Qiao, Y.; Schelter, E. J. Lanthanide photocatalysis. Acc. Chem. Res. 2018, 51, 2926-2936.

(3) He, Y.; Lopez, A.; Zhang, Z.; Chen, D.; Yang, R.; Liu, J. Nucleotide and DNA coordinated lanthanides: from fundamentals to applications. Coord. Chem. Rev. 2019, 387, 235-248.

(4) Bünzli, J.-C. G. Lanthanide photonics: Shaping the nanoworld. Trends in Chemistry 2019, 1, 751-762.

(5) Aulsebrook, M. L.; Graham, B.; Grace, M. R.; Tuck, K. L. Lanthanide complexes for luminescene-based sensing of low molecular weight analytes. Coord. Chem. Rev. 2018, 375, 191-220.

(6) Terraschke, H.; Wickleder, C. UV, blue, green, yellow, red, and small: newest developments on $\mathrm{Eu}^{2+}$-doped nanophosphors. Chem. Rev. 2015, 115, 11352-11378.

(7) Binnemans, K. Lanthanide-based luminescent hybrid materials. Chem. Rev. 2009, 109, 4283-4374.

(8) Dorenbos, P. The quest for high resolution $\gamma$-ray scintillators. Opt. Mater. 2019, 1, 100021.

(9) Li, G.; Tian, Y.; Zhao, Y.; Lin, J. Recent progress in luminescence tuning of $\mathrm{Ce}^{3+}$ and $\mathrm{Eu}^{2+}$-activated phosphors for pc-WLEDs. Chem. Soc. Rev. 2015, 44, 8688-8713.

(10) Horky, K.; Schnick, W. $\mathrm{Li}_{24} \mathrm{Sr}_{12}\left[\mathrm{Si}_{24} \mathrm{~N}_{47} \mathrm{O}\right] \mathrm{F}: \mathrm{Eu}^{2+}$ Structure and luminescence of an orange phosphor. Chem. Mater. 2017, 29, 45904596.

(11) Aiga, F.; Hiramatsu, R.; Ishida, K. Ab initio theoretical study of $4 f-5 d$ transitions in $\mathrm{Eu}^{2+}$-doped $\mathrm{CaF}_{2}$ : (2) augmented-basis-set-study. J. Lumin. 2016, 169, 601-605.

(12) Ning, L.; Huang, X.; Huang, Y.; Tanner, P. A. Origin of the green persistent luminescence of Eu-doped $\mathrm{SrAl}_{2} \mathrm{O}_{4}$ from a multiconfigurational ab initio study of $4 f^{7}-4 f^{6} 5 d^{1}$ transitions. J. Mater. Chem. C 2018, 6, 6637-6640.

(13) Faucher, M. D.; Moune, O. K. $4 f^{2} / 4 f 6 p$ configuration interaction in $\mathrm{LiYF}_{4}: \mathrm{Pr}^{3+}$. Phys. Rev. A: At., Mol., Opt. Phys. 1997, $55,4150$.

(14) Marx, R.; Moro, F.; Dörfel, M.; Ungur, L.; Waters, M.; Jiang, S. D.; Orlita, M.; Taylor, J.; Frey, W.; Chibotaru, L. F.; van Slageren, K. Spectroscopic determination of crystal field splittings in lanthanide double deckers. Chem. Sci. 2014, 5, 3287-3293.

(15) Cimpoesu, F.; Frecus, B.; Oprea, C. I.; Ramanantoanina, H.; Urland, W.; Daul, C. On exchange coupling and bonding in the $\mathrm{Gd}_{2} @$ $\mathrm{C}_{80}$ and $\mathrm{Gd}_{2} @ \mathrm{C}_{79} \mathrm{~N}$ endohedral dimetallo-fullerenes. Mol. Phys. 2015, 113, 1712-1727.

(16) Cimpoesu, F.; Dragoe, N.; Ramanantoanina, H.; Urland, W.; Daul, C. The theoretical account of the ligand field bonding regime and magnetic anisotropy in the $\mathrm{DySc}_{2} \mathrm{~N} @ \mathrm{C}_{80}$ single ion magnet endohedral fullerene. Phys. Chem. Chem. Phys. 2014, 16, 1133711348.

(17) Jung, J.; Islam, M. A.; Pecoraro, V. L.; Mallah, T.; Berthon, C.; Bolvin, H. Derivation of lanthanide series crystal field parameters from first principles. Chem. - Eur. J. 2019, 25, 15112.

(18) Aravena, D.; Atanasov, M.; Neese, F. Periodic trends in lanthanide compounds through the eyes of multireference $a b$ initio theory. Inorg. Chem. 2016, 55, 4457-4469.

(19) Armelao, L.; Quici, S.; Barigelletti, F.; Accorsi, G.; Bottaro, G.; Cavazzini, M.; Tondello, E. Desing of luminescent lanthanide complexes: from molecules to highly efficient photo-emitting materials. Coord. Chem. Rev. 2010, 254, 487-505. 
(20) Kawasaki, K.; Sugiyama, R.; Tsuji, T.; Iwasa, T.; Tsunoyama, H.; Mizuhata, Y.; Tokitoh, N.; Nakajima, A. A designer ligand field for blue-green luminescence of organoeuropium(II) sandwich complexes with cyclononatetraenyl ligands. Chem. Commun. 2017, 53, 65576560.

(21) Atanasov, M.; Daul, C.; Rauzy, C. A DFT based ligand field theory. Struct. Bonding (Berlin, Ger.) 2004, 106, 97.

(22) Ramanantoanina, H.; Urland, W.; Cimpoesu, F.; Daul, C. Ligand field density functional theory calculation of the $4 f^{2}-4 f^{1} 5 d^{1}$ transitions in the quantum cutter $\mathrm{Cs}_{2} \mathrm{KYF}_{6}: \mathrm{Pr}^{3+}$. Phys. Chem. Chem. Phys. 2013, 15, 13902-13910.

(23) Ramanantoanina, H.; Sahnoun, M.; Barbiero, A.; Ferbinteanu, M.; Cimpoesu, F. Development and applications of the LFDFT: the non-empirical account of ligand field and the simulation of the $f-d$ transitions by density functional theory. Phys. Chem. Chem. Phys. 2015, 17, 18547-18557.

(24) Salkeld, A. J.; Reid, M. F.; Wells, J.-P. R.; Sanchez-Sanz, G.; Seijo, L.; Barandiaran, Z. Effective Hamiltonian parameters for $a b$ initio energy-level calculations of $\mathrm{SrCl}_{2}: \mathrm{Yb}^{2+}$ and $\mathrm{CsCaBr}_{3}: \mathrm{Yb}^{2+}$. J. Phys.: Condens. Matter 2013, 25, 415504.

(25) Ogasawara, K.; Watanabe, S.; Toyoshima, H.; Ishii, T.; Brik, M. G.; Ikeno, H.; Tanaka, I. Optical spectra of trivalent lanthanides in $\mathrm{LiYF}_{4}$ crystal. J. Solid State Chem. 2005, 178, 412-418.

(26) Dorenbos, P. Systematic behavior in trivalent lanthanide charge transfer energies. J. Phys.: Condens. Matter 2003, 15, 8417.

(27) McNamara, J. P.; Berrigan, S. D.; Hillier, I. H. Semiempirical molecular orbital scheme to study lanthanide(III) complexes: PM3 parameters for europium, gadolinium and ytterbium. J. Chem. Theory Comput. 2007, 3, 1014-1027.

(28) Sorace, L.; Benelli, C.; Gatteschi, D. Lanthanides in molecular magnetism: old tools in a new field. Chem. Soc. Rev. 2011, 40, 30923104.

(29) te Velde, G.; Bickelhaupt, F. M.; Baerends, E. J.; Fonseca Guerra, C.; van Gisbergen, S. J. A.; Snijders, G.; Ziegler, T. Chemistry with ADF. J. Comput. Chem. 2001, 22, 931-967.

(30) Fonseca Guerra, C.; Snijders, J. G.; te Velde, G.; Baerends, E. J. Towards an order-N DFT method. Theor. Chem. Acc. 1998, 99, 391403.

(31) Baerends, E. J.; Ziegler, T.; Atkins, A. J.; Autschbach, J.; Baseggio, O.; Bashford, D.; Berces, A.; Bickelhaupt, F. M.; Bo, C.; Boerrigter, P. M.; Cavallo, L.; Daul, C.; Chong, D. P.; Chulhai, D. V.; Deng, L.; Dickson, R. M.; Dieterich, J. M.; Ellis, D. E.; van Faassen, M.; Fan, L.; Fischer, T. H.; Fonseca Guerra, C.; Franchini, M.; Ghysels, A.; Giammona, A.; van Gisbergen, S. J. A.; Goez, A.; Götz, A. W.; Groeneveld, J. A.; Gritsenko, O. V.; Grüning, M.; Gusarov, S.; Harris, F. E.; van den Hoek, P.; Hu, Z.; Jacob, C. R.; Jacobsen, H.; Jensen, L.; Joubert, L.; Kaminski, J. W.; van Kessel, G.; König, C.; Kootstra, F.; Kovalenko, A.; Krykunov, M. V.; van Lenthe, E.; McCormack, D. A.; Michalak, A.; Mitoraj, M.; Morton, S. M.; Neugebauer, J.; Nicu, V. P.; Noodleman, L.; Osinga, V. P.; Patchkovskii, S.; Pavanello, M.; Peeples, C. A.; Philipsen, P. H. T.; Post, D.; Pye, C. C.; Ramanantoanina, H.; Ramos, P.; Ravenek, W.; Rodriguez, J. I.; Ros, P.; Rüger, R.; Schipper, P. R. T.; Schlüns, D.; van Schoot, H.; Schreckenbach, G.; Seldenthuis, J. S.; Seth, M.; Snijders, J. G.; Sola, M.; Stener, M.; Swart, M.; Swerhone, D.; Tognetti, V.; te Velde, G.; Vernooijs, P.; Versluis, L.; Visscher, L.; Visser, O.; Wang, F.; Wesolowski, T. A.; van Wezenbeek, E. M.; Wiesenekker, G.; Wolff, S. K.; Woo, T. K.; Yakovlev, A. L. ADF2019. http://www.scm.com. (2019)

(32) Ramanantoanina, H.; Daul, C. A non-empirical calculation of $2 p$ core-electron excitation in compounds with $3 d$ transition metal ions using ligand-field and density functional theory (LFDFT). Phys. Chem. Chem. Phys. 2017, 19, 20919-20929.

(33) Ramanantoanina, H. A DFT-based theoretical model for the calculation of spectral profiles of lanthanide $\mathrm{M}_{4,5}$-edge $\mathrm{x}$-ray absorption. J. Chem. Phys. 2018, 149, 054104.

(34) Ziegler, T.; Rauk, A.; Baerends, E. J. On the calculation of multiplet energies by the Hartree-Fock-Slater method. Theor. Chem. Acc. 1977, 43, 261-271.
(35) Endicott, J. F.; Watzky, M. A.; Song, X.; Buranda, T. Observations implicating vibronic coupling in covalently linked transition metal electron transfer systems. Coord. Chem. Rev. 1997, $159,295-323$.

(36) Bersuker, I. B.; Polinger, V. Z. Vibronic interactions in molecules and crystals; Springer-Verlag: Berlin, 1989.

(37) Bersuker, I. B. The Jahn-Teller effect and vibronic interactions in modern chemistry; Springer: New York, 1984.

(38) Vosko, S. H.; Wilk, L.; Nusair, M. Accurate spin-dependent electron liquid correlation energies for local spin density calculations: a critical analysis. Can. J. Phys. 1980, 58, 1200-1211.

(39) Perdew, J.; Burke, K.; Ernzerhof, M. Generalized gradient approximation made simple. Phys. Rev. Lett. 1996, 77, 3865.

(40) Becke, A. D. Density-functional thermochemistry. III. The role of exact exchange. J. Chem. Phys. 1993, 98, 5648.

(41) Grimme, S.; Antony, J.; Ehrlich, S.; Krieg, H. A consistent and accurate $\mathrm{ab}$ initio parameterization of density functional dispersion correction (DFT-D) for the 94 elements H-Pu. J. Chem. Phys. 2010, $132,154104$.

(42) Sénéchal-David, K.; Hemeryck, A.; Tancrez, N.; Toupet, L.; Williams, J. A. G.; Ledoux, I.; Zyss, J.; Boucekkine, A.; Guégan, J.-P.; Le Bozec, H.; Maury, O. Synthesis, structural studies, theoretical calculations, and linear and nonlinear optical properties of terpyridyl lanthanide complexes: new evidence for the contribution of $f$ electrons to the NLO activity. J. Am. Chem. Soc. 2006, 128, 12243-12255.

(43) Tancrez, N.; Feuvrie, C.; Ledoux, I.; Zyss, J.; Toupet, L.; Le Bozec, H.; Maury, O. Lanthanide complexes for second order nonlinear optics: evidence for the direct contribution of $f$ electrons to the quadratic hyperpolarizability. J. Am. Chem. Soc. 2005, 127, 13474-13475.

(44) Schinzel, S.; Bindl, M.; Visseaux, M.; Chermette, H. Structural and electronic analysis of lanthanide complexes: reactivity may not necessarily be indepdendent of the identity of the lanthanide atom a DFT study. J. Phys. Chem. A 2006, 110 (39), 11324-11331.

(45) van Lenthe, E.; Baerends, E. J.; Snijders, J. G. Relativistic regular two-component Hamiltonians. J. Chem. Phys. 1993, 99, 4597. (46) We have to keep in mind that LFDFT calculation uses a parametrization scheme that involves the Slater-Condon integrals and spin-orbit coupling constants, and the ligand-field potential acts as a weak perturbation. Hence, the parameters are less concerned with the change of the molecular symmetry $\left(D_{9}\right.$ or $D_{9 d}$ or $\left.D_{9 h}\right)$ in the present calculation. More particularly, we find that only the diagonal element of the ligand-field potential is nonzero by symmetry, even in $D_{9}$ where mixing between $4 \mathrm{f}$ and $5 \mathrm{~d}$ orbitals are foreseen (by having $4 \mathrm{f}$ and $5 \mathrm{~d}$ components that share similar representations $\mathrm{e}_{1}$ and $\mathrm{e}_{2}$; see Table 3).

(47) Xemard, M.; Zimmer, S.; Cordier, M.; Goudy, V.; Ricard, L.; Clavaguéra, C.; Nocton, G. Lanthanidocenes: synthesis, structure, and bonding of linear sandwich complexes of lanthanides. J. Am. Chem. Soc. 2018, 140, 14433-14439.

(48) Chang, A. H. H.; Pitzer, R. M. Electronic structure and spectra of uranocene. J. Am. Chem. Soc. 1989, 111, 2500-2507.

(49) Hayes, R. G.; Edelstein, N. An elementary molecular orbital calculation on $\mathrm{U}\left(\mathrm{C}_{8} \mathrm{H}_{8}\right)_{2}$ and its application to the electronic structures of $\mathrm{U}\left(\mathrm{C}_{8} \mathrm{H}_{8}\right)_{2}, \mathrm{~Np}\left(\mathrm{C}_{8} \mathrm{H}_{8}\right)_{2}$ and $\mathrm{Pu}\left(\mathrm{C}_{8} \mathrm{H}_{8}\right)_{2}$. J. Am. Chem. Soc. 1972, 94, 8688-8691.

(50) Li, J.; Bursten, B. E. Relativistic density functional study of the geometry, electronic transitions, ionization energies and vibrational frequencties of protactinocene, $\mathrm{Pa}\left(\mathrm{C}_{8} \mathrm{H}_{8}\right)_{2}$. J. Am. Chem. Soc. 1998, 120, 11456-11466.

(51) Boerrigter, P. M.; Baerends, E. J.; Snijders, J. G. A relativistic LCAO Hartree-Fock-Slater investigation of the electronic structure of the actinocene $\mathrm{M}(\mathrm{COT})_{2}, \mathrm{M}=\mathrm{Th}, \mathrm{Pa}, \mathrm{U}, \mathrm{Np}$ and $\mathrm{Pu}$. Chem. Phys. 1988, 122, 357-374.

(52) Ramanantoanina, H.; Gruden, M. Theoretical insight into the magnetic circular dichroism of uranium $\mathrm{N}_{6,7}$-edge, X-ray absorption. Int. J. Quantum Chem. 2019, DOI: 10.1002/qua.26081. 
(53) Gendron, F.; Pritchard, B.; Bolvin, H.; Autschbach, J. Single-ion $4 f$ element magnetism: an ab-initio look at $\operatorname{Ln}(\mathrm{COT})_{2}{ }^{-}$. Dalton Trans 2015, 44, 19886-19900.

(54) Kurikawa, T.; Negishi, Y.; Hayakawa, F.; Nagao, S.; Miyajima, K.; Nakajima, A.; Kaya, K. Multiple-decker sandwich complexes of lanthanide-1,3,5,7-cyclooctatetraene $\left[\operatorname{Ln}_{\mathrm{n}}\left(\mathrm{C}_{8} \mathrm{H}_{8}\right)_{\mathrm{m}}\right](\mathrm{Ln}=\mathrm{Ce}, \mathrm{Nd}, \mathrm{Eu}$, $\mathrm{Ho}$ and $\mathrm{Yb}$ ); localized ionic bonding structure. J. Am. Chem. Soc. 1998, 120 (45), 11766-11772.

(55) Hodgson, K. O.; Mares, F.; Starks, D. F.; Streitwieser, A. Lanthanide (III) complexes with cyclooctatetraene dianion. Synthetic chemistry, characterization and physical properties. J. Am. Chem. Soc. 1973, 95, 8650-8658.

(56) Hannachi, D.; Haroun, M. F.; Khireddine, A.; Chermette, H. Optical and nonlinear optical properties of $\operatorname{Ln}(\mathrm{Tp})_{2}$, where $\mathrm{Ln}=\mathrm{La}$, ..., Lu and $\mathrm{Tp}=$ tris(pyrazolyl)borate: DFT+TD-DFT study. New J. Chem. 2019, 43, 14377-14389.

(57) Ramanantoanina, H.; Cimpoesu, F.; Göttel, C.; Sahnoun, M.; Herden, B.; Suta, M.; Wickleder, C.; Urland, W.; Daul, C. Prospecting lighting applications with ligand field tools and density functional theory: a first-principles account of the $4 f^{7}-4 f^{6} 5 d^{1}$ luminescence of $\mathrm{CsMgBr}: \mathrm{Eu}^{2+}$. Inorg. Chem. 2015, 54, 8319-8326.

(58) Jørgensen, C. K. Modern aspects of ligand-field theory; NorthHolland Publishing Co.: Amsterdam, 1971.

(59) Figgis, B. N.; Hitchman, M. A. Ligand field theory and its applications; Wiley-VCH: New York, 2000.

(60) Ramanantoanina, H. On the calculation of multiplet energies of three-open-shell $4 f^{13} 5 f^{\mathrm{n}} 6 d^{1}$ electron configuration by LFDFT: modeling the optical spectra of $4 f$ core-electron excitation in actinide compounds. Phys. Chem. Chem. Phys. 2017, 19, 32481-32491.

(61) Ramanantoanina, H.; Studniarek, M.; Daffé, N.; Dreiser, J. Non-empirical calculation of X-ray magnetic circular dichroism in lanthanide compounds. Chem. Commun. 2019, 55, 2988-2991.

(62) Ramanantoanina, H.; Kuri, G.; Daul, C.; Bertsch, J. Core electron excitations in $\mathrm{U}^{4+}$ : modeling of the $\mathrm{n} d^{10} 5 f^{2}-\mathrm{n} d^{9} 5 f^{3}$ transitions with $\mathrm{n}=3,4$ and 5 by ligand field tools and density functional theory. Phys. Chem. Chem. Phys. 2016, 18, 19020-19031.

(63) Ramanantoanina, H.; Kuri, G.; Martin, M.; Bertsch, J. Study of electronic structure in the L-edge spectroscopy of actinide materials: $\mathrm{UO}_{2}$ as an example. Phys. Chem. Chem. Phys. 2019, 21, 7789-7801.

(64) Suta, M.; Wickleder, C. Synthesis, spectroscopic properties and applications of divalent lanthanides apart from $\mathrm{Eu}^{2+}$. J. Lumin. 2019, 210, 210-238.

(65) Petrakis, L. Spectral line shapes: gaussian and Lorentzian functions in magnetic resonance. J. Chem. Educ. 1967, 44, 432.

(66) Gritsenko, O. V.; Schipper, P. R. T.; Baerends, E. J. Approximation of the exchange-correlation Kohn-Sahm potential with a statistical average of different orbital model potentials. Chem. Phys. Lett. 1999, 302, 199-207.

(67) Schipper, P. R. T.; Gritsenko, O. V.; van Gisbergen, S. J. A.; Baerends, E. J. Molecular calculations of excitation energies and hyperpolarizabilities with a statistical average of orbital model exchange-correlation potentials. J. Chem. Phys. 2000, 112, 1344.

(68) Klan, P.; Wirz, J. Photochemistry of organic compounds from concepts to practice; Wiley: West Sussex, 2009.

(69) de Jong, M.; Seijo, L.; Meijerink, A.; Rabouw, F. T. Resolving the ambiguity in the relation between Stokes shift and Huang-Rhys parameter. Phys. Chem. Chem. Phys. 2015, 17, 16959-16969.

(70) Ruhoff, P. T.; Ratner, M. A. Algorithms for computing FranckCondon overlap integrals. Int. J. Quantum Chem. 2000, 77, 383-392. (71) Dushinsky, F. The importance of the electron spectrum in multi atomic molecules concerning the Franck-Condon principle [in russian]. Acta Physicochim. URSS 1937, 7, 551-566.

(72) $R_{\mathrm{ES}}$ and $R_{\mathrm{GS}}$ are column vectors that contain $3 N$ elements $(N$ being the total number of atoms in the molecule, i.e., 37) of the Cartesian coordinates corresponding to the optimized geometries of $\mathrm{Eu}\left(\eta^{9}-\mathrm{C}_{9} \mathrm{H}_{9}\right)_{2}$ with Eu configurations $4 \mathrm{f}^{6} 5 \mathrm{~d}^{1}$ and $4 \mathrm{f}^{7}$, respectively. See also: Ramanantoanina, H.; Zlatar, M.; Garcia-Fernandez, P.; Daul, C.; Gruden-Pavlovic, M. General treatment of the multimode Jahn-Teller effect: study of fullerene cations. Phys. Chem. Chem. Phys. 2013, 15, $1252-1259$.

(73) Moreno, M.; Barriuso, M. T.; Aramburu, J. A. The Huang-Rhys factor $S\left(\mathrm{a}_{1 \mathrm{~g}}\right)$ for transition metal impurities: a microscopic insight. $J$. Phys.: Condens. Matter 1992, 4, 9481-9488.

(74) Henderson, B.; Imbusch, G. F. Optical spectroscopy of inorganic solids; Clarendon Press: Oxford, 1989. 